\title{
Investigation of Hypersonic Laminar Heating Augmentation in the Stagnation Region
}

\author{
Eric C. Marineau ${ }^{1}$, Daniel R. Lewis ${ }^{2}$, Michael S. Smith ${ }^{3}$, John F. Lafferty ${ }^{4}$ \\ AEDC White Oak, Silver Spring, MD, 20903 \\ Molly E. White ${ }^{5}$ and Adam J. Amar ${ }^{6}$ \\ NASA Lyndon B. Johnson Space Center, Houston, TX, 77058
}

\begin{abstract}
Laminar stagnation region heating augmentation is investigated in the AEDC Tunnel 9 at Mach 10 by performing high frequency surface pressure and heat transfer measurements on the Orion CEV capsule at zero degree angle-of-attack for unit Reynolds numbers between 0.5 and 15 million per foot. Heating augmentation increases with Reynolds number, but is also model size dependent as it is absent on a 1.25-inch diameter model at Reynolds numbers where it reaches up to $15 \%$ on a 7 -inch model. Heat transfer space-time correlations on the 7-inch model show that disturbances convect at the boundary layer edge velocity and that the streamwise integral scale increases with distance. Therefore, vorticity amplification due to stretching and piling-up in the stagnation region appears to be responsible for the stagnation point heating augmentation on the larger model. This assumption is reinforced by the $f^{-11 / 3}$ dependence of the surface pressure spectrum compared to the $f^{-1}$ dependence in the free steam. Vorticity amplification does not occur on the 1.25inch model because the disturbances are too large. Improved free stream fluctuation measurements will be required to determine if significant vorticity is present upstream or mostly generated behind the bow shock.
\end{abstract}

\section{Nomenclature}

$c_{p} \quad=$ specific heat of test gas $(\mathrm{J} / \mathrm{kg} / \mathrm{K})$

$D \quad=$ maximum vehicle diameter $(\mathrm{m})$

$\mathrm{f} \quad=$ frequency $(\mathrm{Hz})$

$H_{0} \quad=$ total enthalpy $(\mathrm{J} / \mathrm{kg})$

$H_{300 K} \quad=$ cold-wall $($ at $300 \mathrm{~K})$ enthalpy $(\mathrm{J} / \mathrm{kg})$

$H_{w} \quad=$ enthalpy at measured wall temperature $(\mathrm{J} / \mathrm{kg})$

$\mathrm{L}=$ turbulent length scale

$M_{\infty} \quad=$ free stream Mach number

$M_{e} \quad=$ boundary-layer edge Mach number

$p_{\infty} \quad=$ free stream pressure $(\mathrm{Pa})$

$\operatorname{Pr} \quad=$ Prandtl number

$q \quad=$ heat transfer rate $\left(\mathrm{W} / \mathrm{m}^{2}\right)$

$\mathrm{R}=\quad$ maximum vehicle radius $(\mathrm{m})$

$\mathrm{R}_{\mathrm{n}}=\quad=$ nose (spherical cap) radius (m)

$\mathrm{R}_{\mathrm{T}}=\quad=$ radius at tangency point of spherical cap and shoulder $(\mathrm{m})$

$\mathrm{R}_{\mathrm{S}}=\quad=$ radius at aftbody shoulder $(\mathrm{m})$

$\mathrm{Re}=\quad=$ free stream unit Reynolds Number $(1 / \mathrm{m}$ or $1 / \mathrm{ft})$

$\operatorname{Re}_{\mathrm{D}}=\quad=$ free stream Reynolds Number based on diameter

${ }^{1}$ Lead Aerospace Technologist, AEDC White Oak, Silver Spring, MD, Member AIAA.

${ }^{2}$ Project Engineer, Aerospace Testing Alliance, Silver Spring, MD, Member AIAA.

${ }^{3}$ Senior Research Engineer, Aerospace Testing Alliance, Silver Spring, MD, Senior Member AIAA.

${ }^{4}$ Technical Director, AEDC White Oak, Silver Spring, MD, Associate Fellow AIAA.

${ }^{5}$ Aerospace Engineer, Applied Aeroscience and CFD Branch, Mail Code EG3, Member AIAA.

${ }^{6}$ Aerospace Engineer, Applied Aeroscience and CFD Branch, Mail Code EG3, Member AIAA. 


$\begin{array}{ll}\mathrm{St}= & =\text { Stanton number } \\ \mathrm{Stx} \times\left(\operatorname{Re}_{\mathrm{D}}\right)^{1 / 2} & =\text { correlation parameter for laminar heating data } \\ \mathrm{T}_{\mathrm{w}} & =\text { model wall temperature }(\mathrm{K}) \\ \mathrm{T}_{\infty} & =\text { free stream temperature }(\mathrm{K}) \\ \mathrm{Tu} & =\text { Turbulence intensity } \\ \mathrm{U}_{\infty} & =\text { free stream velocity }(\mathrm{m} / \mathrm{s}) \\ \mathrm{x}, \mathrm{y}, \mathrm{z} & =\text { vehicle geometric coordinate system variables } \\ \alpha & =\text { angle of attack }(\mathrm{deg}) \\ \delta & =\text { boundary layer thickens } \\ \rho_{\infty} & =\text { free stream density }\left(\mathrm{kg} / \mathrm{m}^{3}\right) \\ \mu_{\infty} & =\text { free stream viscosity }(\mathrm{kg} / \mathrm{m} / \mathrm{s})\end{array}$

\section{Introduction}

Stagnation point heating augmentation has been observed in wind tunnel testing during the Apollo program. $\checkmark$ According to Bertin ${ }^{(1)}$, experimentally determined convective-heat-transfer rates on the Apollo Command Module were between 20 and 80 percent greater than the theoretical values. Similar observations have been made in recent experiments on reentry capsules in the hypersonic regime for both low ${ }^{(2)(3)(4)}$ and high enthalpy hypersonic flows ${ }^{(5)(6)(7)(8)}$. Heat transfer measurements in the stagnation region were found to surpass numerical predictions by factors often exceeding 100 percent in high enthalpy flows and up to 40 percent in cold flows. For reentry capsules at angles attack, the stagnation point augmentation manifests itself as a bump above the numerical prediction near the stagnation point. Much better agreement is generally obtained away from the stagnation point ${ }^{(2)}{ }^{(3)}$ (4). At zero angle of attack, the size of the stagnation region increases as the flow remains subsonic up to the shoulder and at high Reynolds numbers; augmented heating can be observed over the entire heat shield. Measurements in low enthalpy flows have shown that the stagnation point heating augmentation increases with Reynolds number. For high enthalpy measurements, enthalpy effects are also present as for a fixed Reynolds number, the stagnation point heating augmentation increases with enthalpy. In high enthalpy facilities such as reflected shock tunnels, the free stream enthalpy is typically characterized by heat transfer measurements on small spheres or hemispheres. Such measurements in various shock tunnels ${ }^{(9)(10)(11)}$ at enthalpies between 10 and $20 \mathrm{MJ} / \mathrm{kg}$ on probes with noise Radii between 10 and $20 \mathrm{~mm}$ have shown good agreement with computations ${ }^{(12)}{ }^{(9)}$ for free stream conditions where heating augmentation was measured on larger diameter reentry capsules. It was therefore postulated that the heating augmentation could be model size dependent. However, since $R e_{D}$ was much smaller on the probes compared to larger models, Reynolds number effects cannot be ruled out.

Various reasons such as fine or large particulates ${ }^{(13)}$, acoustic disturbances ${ }^{(14)}$ and free stream vorticity ${ }^{(15)(15)(16)}$

(17) have been proposed to explain the stagnation region heating augmentation in hypersonic flows. Existing measurement have focused on mean values of heat transfer either because of instrumentation limitations ${ }^{(2)(3)(4)}$ or short test times in shock tunnels ${ }^{(5)}$ (6) (7) which make it difficult to obtain reliable fluctuation statistics. In the current study, the combination of high frequency instrumentation and long test times compared to the fluctuations time scale made it possible to obtain a statistical description of the heat transfer and pressure fluctuations in the stagnation region.

The effect of model scale was investigated by using 1.25-inch and 7-inch diameter models at a similar $R e_{D}$ value. The ultimate goal was to provide additional data to better understand the augmented heating phenomenon on an existing 7-inch diameter CEV heat-transfer model which was previously tested both in Tunnel 9 and NASA LaRC. High-frequency surface temperature, heat transfer and pressure data were collected on the heat shield in the stagnation region. In addition, high-speed global flowfield imagery was acquired using high speed Schlieren photography. Finally, a new laser induced incandescence (LII) technique was implemented to quantify fine particulate present in the free stream.

The paper is structured as follow. Section II presents background information regarding the heating augmentation in the stagnation region. Section III describes the experimental facility, test articles, instrumentation, data reduction and experimental uncertainties. The computational model used to determine the heating augmentation is presented in Section IV. Section V A. presents the mean heat transfer data and computed heating augmentation. A characterization of small particulates and a statistical description of the effect of large particles are presented in Section V B. and C. respectively. A spectral analysis of the heat transfer and pressure fluctuation is found in Section V D. An analysis of the convective velocity and disturbance scale sizes is found in section V E. Section V F. presents explanations regarding the role of vorticity and the effect of model size on the stagnation point heating augmentation. 


\section{Background}

Various experimental, computation and theoretical studies have quantified the effect of free stream turbulence on the stagnation point heating augmentation in low speed flows. Sutera, Maeder and Kestin ${ }^{(18)}$ have analyzed the effect of a spatially varying velocity fluctuation on the stagnation-point boundary layer flow. The effect on the mean velocity and temperature profiles for the most amplified component, which is parallel to the axis of the cylinder, was calculated. It was shown that vorticity amplification due to stretching of vortex filaments in the strongly diverging stagnation-point flow is the essential mechanism for the increased heat transfer in the stagnation region for flow with free stream vorticity. The added vorticity increases both the momentum and heat transfer at the wall, but the effect on the heat transfer is up to 10 times greater than the effect on the shear stress. The analysis was limited to disturbances of the scale of the stagnation point boundary layer thickness. For such scales, it was found that vorticity components greater than a neutral wavelength amplify more rapidly than they are dissipated. Sadeh, Sutera \& Maeder ${ }^{(19)}$ extended the analysis to the outer flow showing that small-scale disturbances decay upstream of the stagnation point before being amplified close to the stagnation point.

The theory of Sutera et al. was applied by Weeks ${ }^{(16)(17)}$ to quantify the effect of free stream turbulence on the hypersonic stagnation zone heating. The incompressible model was assumed to be valid, because of the low Mach number in the stagnation region which implies that compressibility effects are small. Qualitative agreement was obtained with heat transfer measurements on a small probe inside a turbulent hypersonic boundary layer where the stagnation point heating reached 2.25 times the laminar value. Quantitative agreement could not be achieved because of large scatter in the experimental data. Free steam turbulence has also been identified by Hoshizaki et al. ${ }^{(15)}$ as a credible mechanism for stagnation point heat transfer enhancement in arc heated facilities. Hoshizaki et al. compiled experimental data obtained in subsonic flows for stagnation point heating on cylinders and spheres subjected to free stream turbulence. The heating augmentation data was correlated as a function $T u R e_{D}$ for spheres and $T u R e_{D}{ }^{0.5}$ for cylinders. Sphere data showed that significant enhancement in heat transfer is obtained when $T u R e_{D}$ is greater than 10E4 showing that in high Reynolds Number flows, a very small amount of turbulence can have a significant impact on the heat transfer. The effect of turbulent scale sizes was not discussed in details as the experimental data considered was for a very limited range of scale sizes. Analytical studies performed by Traci and Wilcox ${ }^{(20)}$ based on the Saffman turbulence model and the use of analytical/numerical solution matching showed a limited effect of scale sizes on heat transfer for the $L / D R e_{D}{ }^{0.5}$ between 10 and 100 . Values of $L / D R e_{D}{ }^{0.5}$ larger than 100 were not considered.

To better understand the effect of length scale on vorticity amplification, Britter, Hunt and Mumford (21) performed hot wire measurements in grid-generated turbulence past a circular cylinder. Velocity fluctuations at various radial and circumferential positions were measured for ratios of cylinder radius to incident turbulence scale $(a / L)$ ranging from 0.05 to 1.42 . The amplification and reduction of the three components of turbulence is explained qualitatively in terms of the distortion by the mean flow of the turbulent vorticity and the blocking effect caused by turbulence impinging on the surface of the cylinder. The relative importance of distortion over blocking increases as $a / L$ increases or the distance from the surface distance increases. When the ratio $a / L$ is small (large vorticity scale), the blocking mechanism is dominant and extend to a distance approximately $L$ form the cylinder, but when $a / L$ is large (small vorticity scale), vorticity distortion is the dominant process out to a distance approximately $3 \mathrm{a}$ from the cylinder except within a distance $L$ where blocking is comparable to distortion. The experimental measurements were bounded by the prediction from rapid distortion theory $(\mathrm{RDT}){ }^{(22)}$ for the very large and very small scales.

In hypersonic flow, the distance over which distortion can operate is reduced, as the flow cannot sense the presence of the body ahead of the bow shock. Intuitively a given scale will appear larger in hypersonic flow because of the limited region of influence. It seems fair to assume that $L$ must not be significantly larger than the shock standoff distance for vorticity distortion to occur.

Evidence of vorticity stretching in the stagnation region has been obtained by Wei and Miau ${ }^{(23)}$ using autocorrelations between two hot wires with variable spacing. The stretched eddies are predominantly aligned in the streamwise direction and their length scale is function of the integral length scale of the incoming freestream turbulence. The measurements show that inviscid vorticity stretching is responsible for the development of vortical structures in the stagnation region outside of the boundary layer as predicted by RDT.

Dullenkopf and Mayle ${ }^{(24)}$ have identified the importance of the turbulence size with respect to the size of the body for augmented stagnation point heating. A linear relationship was found between the heating augmentation and an "effective" turbulence intensity based on a dominant frequency corresponding to a scale approximately equal to $16 \delta$. Turbulent intensity and Reynolds number alone are therefore not sufficient to characterize the stagnation point heating augmentation such that the turbulent scale size with respect to the scale of the body must be known. 


\section{Experimental Methods}

\section{A. Experimental Facility}

The experiments were conducted in the Air Force's Arnold Engineering Development Center (AEDC) Hypervelocity Wind Tunnel No. 9 (Tunnel 9) depicted in Figure 1. Tunnel 9 located in Silver Spring, Maryland is an hypersonic, nitrogen-gas, blow-down wind tunnel with interchangeable nozzles that allow for testing at Mach numbers of $7,8,10$, and 14 over a $0.054 \times 10^{6} / \mathrm{ft}$ to $48.4 \times 10^{6} / \mathrm{ft}\left(0.177 \times 10^{6} / \mathrm{m}\right.$ to $\left.158.8 \times 10^{6} / \mathrm{m}\right)$ unit Reynolds number range (depending on the nozzle). The test section is a $5 \mathrm{ft}(1.52 \mathrm{~m})$ diameter, $12 \mathrm{ft}(3.66 \mathrm{~m})$ long cell that enables testing of large-scale model configurations. Tunnel 9 features a pitch system that can sweep models from -10 deg to $50 \mathrm{deg}$ at pitch rates up to $80 \mathrm{deg} / \mathrm{s}$. With the tunnel's 0.2 second to 15 seconds run times, the dynamic pitch capability allows for a large volume of data to be captured over an entire range of pitch angles during a single run. A detailed description of the facility can be found in ${ }^{(25)}$.
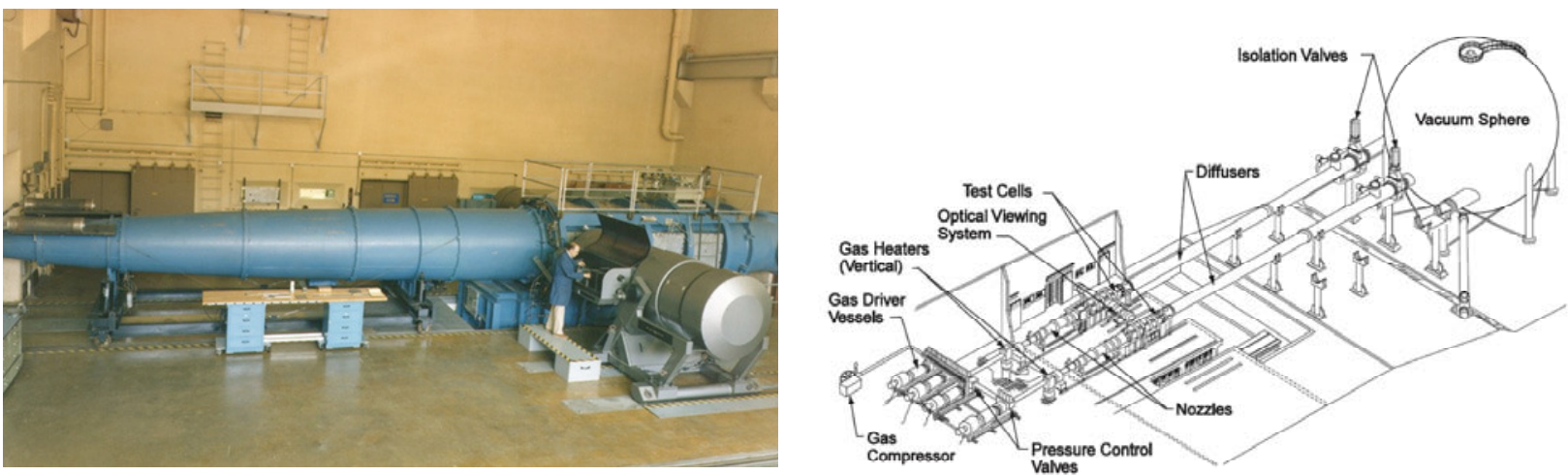

Figure 1. AEDC Tunnel 9 Photograph and Schematic

\section{B. Test Matrix and Conditions}

A total of ten runs were performed in the AEDC Tunnel 9 during the test program. The test matrix and test conditions are found in Table 1 and Table 2. All runs were at a nominal Mach number of 10 for Reynolds numbers between 0.5 and 15 million per foot. Eight runs were with the 7-inch CEV model on the nozzle centerline with four auxiliary probes (PTNT, PTST, PTNB and PTSB) as shown in Figure 2. The auxiliary probes used for each runs are found in Table 1. In addition, two runs were performed with the 1.25-inch Apollo probe on the nozzle centerline to assess the effect radial position on heat transfer.

The supply and Pitot pressure were determined from their respective calibrations performed prior to each run. The supply temperature was determined from the National Institute of Standards and Technology (NIST) tables. The tunnel conditions were calculated from these quantities using a procedure that assumes an isentropic nozzle expansion from the measured supply conditions to the freestream values. An initial estimate for the Mach number is made. Using the estimated Mach number and a measured Pitot pressure, freestream conditions are obtained from perfect gas relations. Using the thermodynamic properties from the standard Mollier diagram for nitrogen and the measured supply conditions, a value of total enthalpy is obtained. A freestream velocity is then obtained based on the conservation of total enthalpy. This value of velocity is converted to Mach number and is compared to the initial estimated Mach number value. When these two agree, the calculation is complete and the tunnel conditions are known. Otherwise, a new value for Mach number is tried and the iteration procedure continues until convergence is reached. This method accounts for high pressure and high temperature effects that occur in the supply area. Note that while the expansion from the nozzle supply area to the test cell is isentropic, it is not calorically perfect, i.e. $\gamma$ is not constant throughout the entire expansion. Therefore, any nozzle expansion calculations completed using the measured nozzle supply conditions should include the appropriate assumptions to account for variable ratio of specific heats for nitrogen through the expansion. 
Table 1: Test Matrix

\begin{tabular}{|c|c|c|c|c|c|c|c|c|c|c|}
\hline Run & Run \# & M & $\begin{array}{c}\mathbf{R e} \\
(\mathbf{E} 6 / \mathbf{f t})\end{array}$ & $\begin{array}{c}\mathbf{R e} \\
(\mathbf{E 6} / \mathbf{m})\end{array}$ & $\begin{array}{c}\alpha \\
(\operatorname{deg})\end{array}$ & $\begin{array}{c}\text { PTNT } \\
\text { Noise Probe } 1\end{array}$ & $\begin{array}{c}\text { PTST } \\
\text { Noise Probe } 2\end{array}$ & PTNB & PTSB & $\begin{array}{l}\text { Usable Test Period } \\
(s)\end{array}$ \\
\hline 1 & 3486 & 10 & 4.0 & 13.1 & 0 & Kulite - 15 psia & PCB & Flat-Face Coax & 1.25" CEV Coax & $0.5-1$ \\
\hline 2 & 3491 & 10 & 2.0 & 6.6 & 0 & Kulite - 15 psia & PCB & Flat-Face ALTP & 1.25" CEV Coax & $0.75-2.25$ \\
\hline 3 & 3492 & 10 & 5.4 & 17.7 & 0 & Kulite - 15 psia & PCB & Flat-Face ALTP & 1.25" CEV Coax & $0.7-1.1$ \\
\hline 4 & 3494 & 10 & 7.0 & 23.0 & 0 & Kulite - 15 psia & PCB & Flat-Face Coax & 1.25" CEV Coax & $0.5-0.9$ \\
\hline 5 & 3495 & 10 & 0.5 & 1.6 & 0 & Kulite - 15 psia & PCB & Flat-Face Coax & 1.25" CEV Coax & $2-5$ \\
\hline 6 & 3496 & 10 & 9.2 & 30.3 & 0 & Kulite - 50 psia & PCB & Flat-Face Coax & 1.25" CEV Coax & $0.4-0.8$ \\
\hline 7 & 3497 & 10 & 14.9 & 48.9 & 0 & Kulite - 50 psia & PCB & Flat-Face Coax & 1.25" CEV Coax & $0.3-0.5$ \\
\hline 8 & 3498 & 10 & 14.9 & 48.9 & 0 & Kulite - 50 psia & PCB & Flat-Face ALTP & 1.5" CEV Coax & $0.3-0.5$ \\
\hline 9 & 3501 & 10 & 9.2 & 30.2 & 0 & $\bar{L}$ & 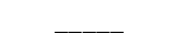 & 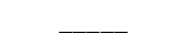 & 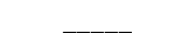 & $0.4-0.8$ \\
\hline 10 & 3502 & 10 & 14.9 & 48.9 & 0 & & & & & $0.3-0.5$ \\
\hline
\end{tabular}

Table 2: Test Conditions

\begin{tabular}{lccccccccc}
\hline \hline Run & $\begin{array}{c}\mathbf{R e} \\
(\mathbf{1 0 E 6} / \mathbf{m})\end{array}$ & $\begin{array}{c}\mathbf{P}_{\mathbf{0}} \\
\mathbf{M P a}\end{array}$ & $\begin{array}{c}\mathbf{T}_{\mathbf{0}} \\
\mathbf{K}\end{array}$ & $\begin{array}{c}\mathbf{H}_{\mathbf{0}} \\
\mathbf{M J} / \mathbf{k g}\end{array}$ & $\begin{array}{c}\mathbf{M}_{\infty} \\
-\end{array}$ & $\begin{array}{c}\mathbf{p}_{\infty} \\
\mathbf{k P a}\end{array}$ & $\begin{array}{c}\mathbf{T}_{\infty} \\
\mathbf{K}\end{array}$ & $\begin{array}{c}\mathbf{\rho}_{\infty} \\
\mathbf{k g} / \mathbf{m} \mathbf{3}^{\wedge}\end{array}$ & $\begin{array}{c}\mathbf{U}_{\infty} \\
\mathbf{m} / \mathbf{s e c}\end{array}$ \\
\hline 3486 & 13.1 & 16.7 & 955.6 & 1.037 & 9.9 & 0.44 & 48.7 & $3.06 \mathrm{E}-02$ & 1404.0 \\
3491 & 6.6 & 8.3 & 955.8 & 1.031 & 9.7 & 0.24 & 50.1 & $1.62 \mathrm{E}-02$ & 1398.2 \\
3492 & 17.7 & 26.9 & 1026.1 & 1.128 & 9.9 & 0.70 & 52.4 & $4.47 \mathrm{E}-02$ & 1465.0 \\
3494 & 23.0 & 30.7 & 1002.1 & 1.103 & 10.0 & 0.78 & 50.6 & $5.17 \mathrm{E}-02$ & 1449.1 \\
3495 & 1.6 & 2.0 & 873.7 & 0.931 & 9.4 & 0.07 & 47.9 & $4.95 \mathrm{E}-03$ & 1327.2 \\
3496 & 30.3 & 43.0 & 1014.9 & 1.129 & 10.1 & 1.07 & 51.0 & $7.06 \mathrm{E}-02$ & 1466.4 \\
3497 & 48.9 & 63.7 & 966.7 & 1.089 & 10.1 & 1.67 & 48.9 & $1.15 \mathrm{E}-01$ & 1440.7 \\
3498 & 48.9 & 67.7 & 999.5 & 1.132 & 10.2 & 1.68 & 49.9 & $1.13 \mathrm{E}-01$ & 1469.2 \\
3501 & 30.2 & 43.7 & 1001.4 & 1.113 & 9.9 & 1.21 & 51.8 & $7.80 \mathrm{E}-02$ & 1455.1 \\
3502 & 48.9 & 68.01 & 985.6 & 1.115 & 10.2 & 1.75 & 49.6 & $1.17 \mathrm{E}-01$ & 1458.2 \\
\hline \hline
\end{tabular}

\section{Test Articles and Instrumentation}

1. 7-Inch CEV Model

The 7-inch diameter NASA CEV was the primary test article for this test program. Model dimensions and instrumentation layout are shown in Figure 3. The test article was fabricated in 2006 to support the first NASA CEV test program at AEDC Tunnel $9^{(2)}$. The CEV test article consists of three main components: sting adaptor, aft cone and heat shield. The sting adaptor and aft cone were retained from the first CEV test entry at Tunnel 9. The existing heat shield was modified by the AEDC machine shop to allow the installation of a variety of surfacemounted sensors. A total of fifteen additional instrumentation holes were machined into the heat shield. Five flush mounted PCB 132A31 pressure gages, five Kulite XT-140, 50 psia pressure gages and five Atomic Layer Thermopile (ALTP) heat transfer gages were installed into the test article. In addition to the surface mounted instrumentation, forty-six Medtherm type-E coaxial thermocouples were retained from the previous test program.

All model surface pressures were recorded using Kulite XT-140, 50 psia pressure transducers. Highfrequency-heat flux measurements were obtained using the ALTP direct-read heat-flux sensor which provides a voltage output proportional to the applied surface heat flux. A thermoelectric field is created when a temperature gradient exist over the yttrium-barium-copper-oxide (YBCO) crystal via the transverse Seebec effect. ${ }^{\text {(26) }}$ A spectral resolution up to several hundreds of kilohertz's is expected as a result of the small thickness of the crystal $(1 \mu \mathrm{m})$ and small sensing area. The manufacturer quotes that a $100 \mathrm{kHz}$ frequency response is obtainable with the ALTP sensor.

A calibration was provided by the manufacturer to convert the measured voltage output to surface heat flux using a scale factor. When using the manufacturer's scale factor, the heat flux magnitude measured with the ALTP sensor did not compare well with the heat flux obtained using coaxial thermocouples. However, the time variation of the ALTP heat flux was in good agreement with the coaxial thermocouples such that a static calibration error or bias 
appears to be present Future plans to obtain a more accurate scale factors for individual ALTP sensors include calibrations in AEDC's Aerothermal Measurements Laboratory. The full length paper will characterize the discrepancy between the ALTP and thermocouple measurements.

Additional heat-transfer and pressure measurements were collected on several smaller auxiliary test articles in order to gain more insight to the augmented heat-transfer phenomena. These auxiliary probes were mounted seventeen inches off centerline as shown in Figure 2. A description of the individual auxiliary probes is provided next.

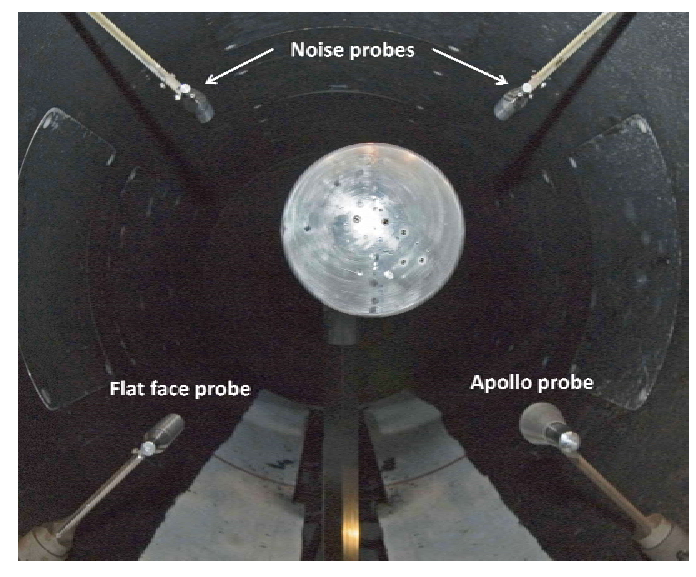

\section{Figure 2. Tunnel 9 Test cell setup}

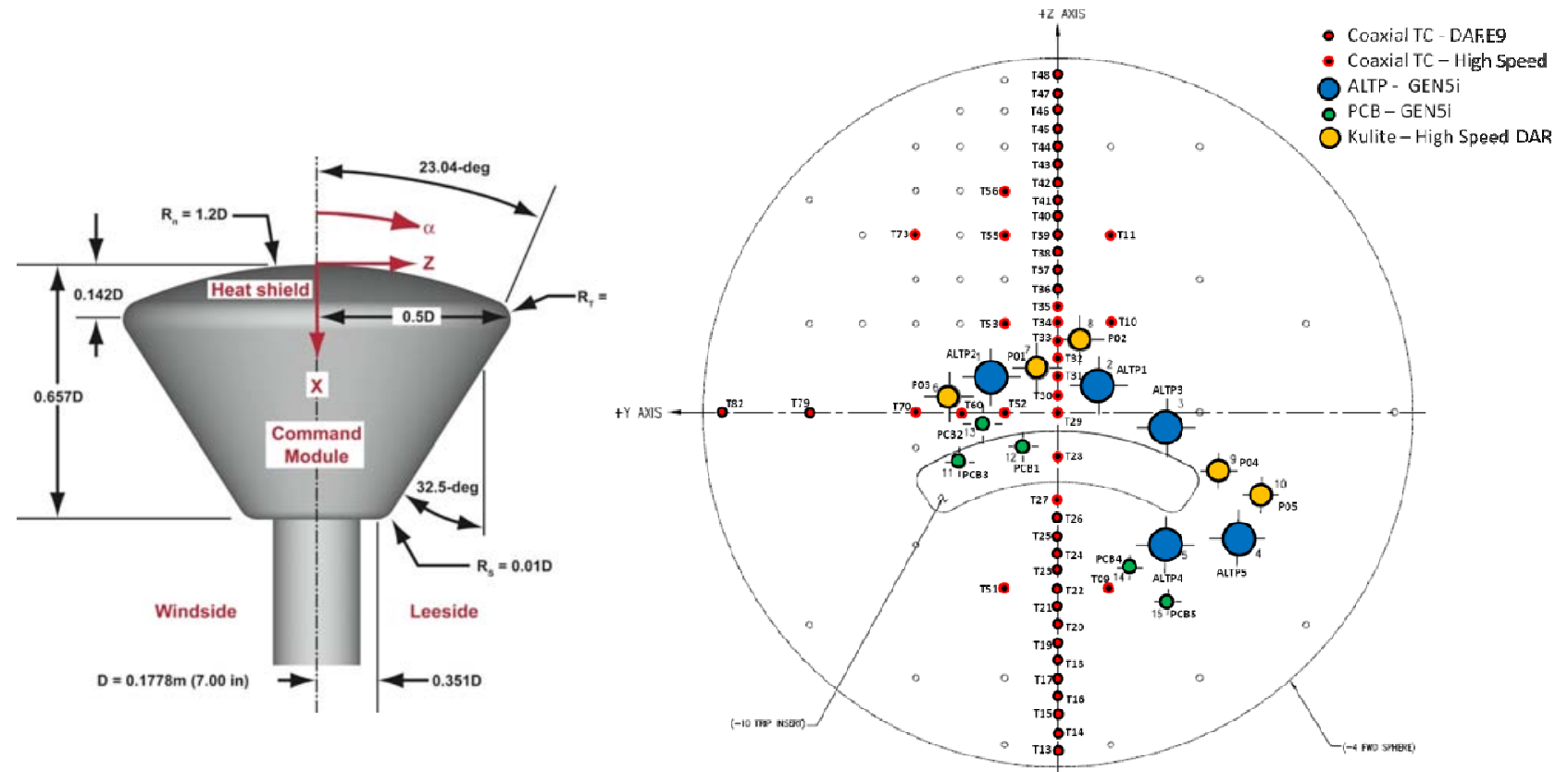

Figure 3. CEV model dimensions left and Instrumentation Layout right

\section{CEV Profile Probes}

To evaluate the effect of size on the stagnation point heating augmentation, a 1.25-inch and a 1.5 -inch diameter replica of the CEV test article was used. The probe was located on the South Bottom (SB) auxiliary sting for 8 runs ( 7 runs for the 1.25 -inch probe). Two additional runs were performed with the 1.25 -inch probe on the nozzle centerline in place of the 7-inch test article. Both the heat shield and corner radius of the probe were scaled based on the dimensions of the primary test article. The 1.25-inch and 1.5-inch CEV probes were both instrumented with seven 0.031-inch Medtherm type-E coaxial thermocouples as shown in Figure 4. 

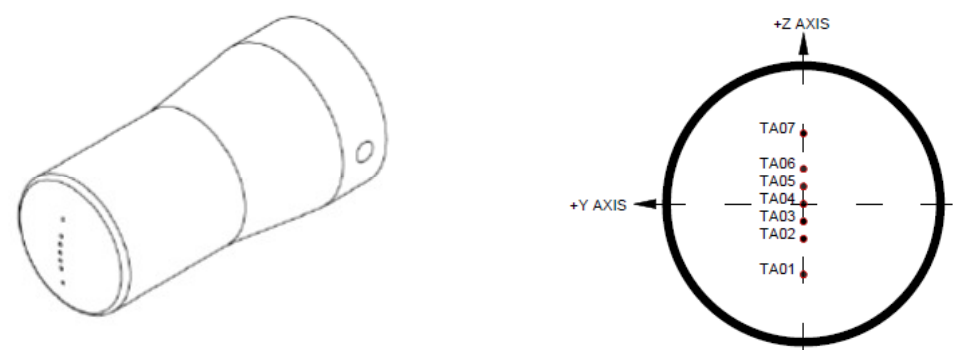

\section{Figure 4. CEV probe (1.25-inch and 1.5 diameters) left and instrumentation layout right}

\section{Flat-Face Probes}

A 0.875 -inch diameter flat-faced cylinder that was mounted perpendicular to the flow. One version of the probe was instrumented with one Medtherm 0.031" type-E coaxial thermocouple and the other with an ALTP sensor as seen in Figure 5. The flat face probe was located on the North Bottom (NB) auxiliary sting for 8 runs; 5 runs using the coaxial thermocouple version and 3 runs using the ALTP version.
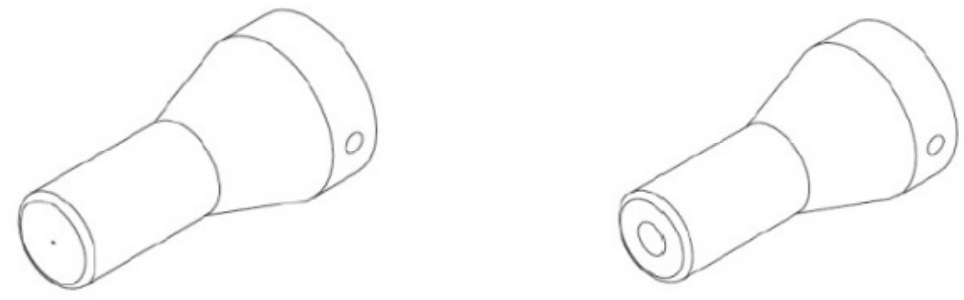

\section{Figure 5. 0.875-inch diameter flat-face probes with thermocouple left and ALTP right}

\section{Noise Probes}

Freestream pressure fluctuation data were collected using two different pressure transducer types. For low frequency measurements, DC-coupled Kulite XCS-093-15A (15 PSI) or XCS-093-50A (50 PSI) absolute pressure transducers, designed for high sensitivity and high signal-to-noise ratio, were used. The gages used included the manufacturer installed "B-screen," which protects the transducer sensing element from potential dust and particulate impacts. However, per manufacturer specification, this B-screen limits the frequency response to $25 \mathrm{kHz}$. ACcoupled PCB 132A31 differential-pressure transducers were used to measure high-frequency freestream pressure fluctuations. The sensing element is a $0.762 \times 0.762 \mathrm{~mm}$ piezoelectric crystal bonded to the sensing surface with conductive epoxy, thus not requiring exposure to the flow through a screen. Per manufacturer specification, these gages have a frequency response range from $11 \mathrm{kHz}$ to over $1 \mathrm{MHz}$. More details about the pressure fluctuation probes and the data reduction are found in Bounitch et al. ${ }^{(27)}$

\section{Flow Visualization}

Schlieren images were acquired at 1,000 and 15,000 frames per second. More details about the Schlieren setup will be available in the paper. Sample frames for run 3498 are shown in Figure 6 along with overlaid CFD solutions. 


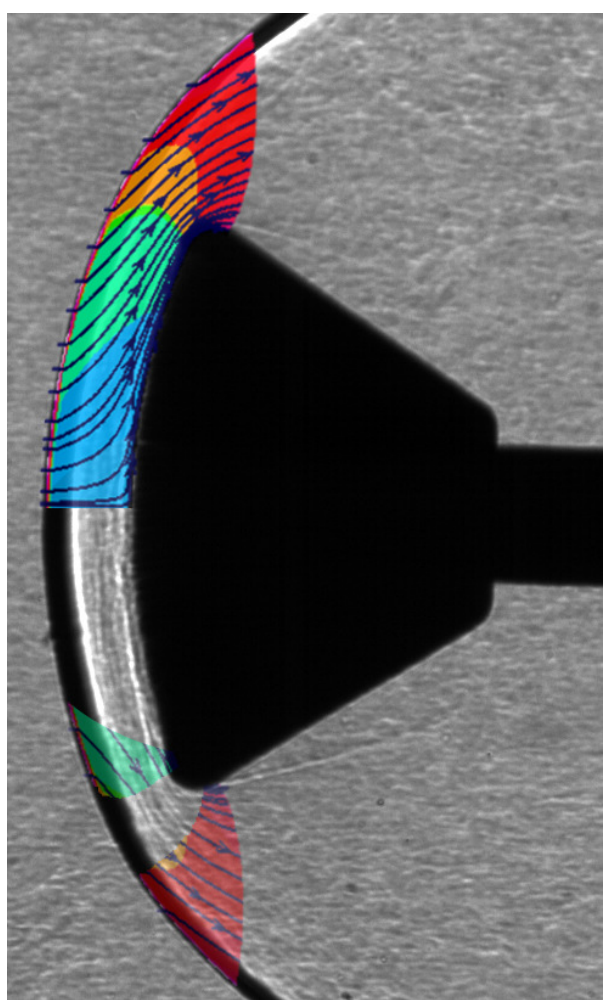

(a) High Resolution $(1 \mathrm{kHz})$

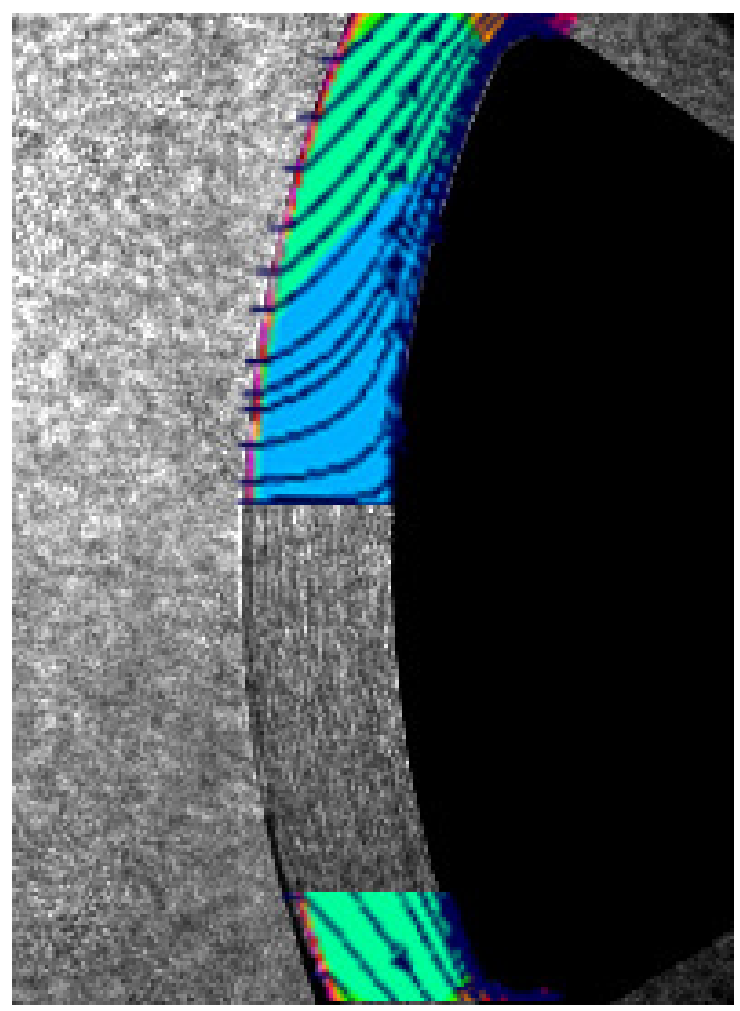

(b) High Speed (15 kHz)

Figure 6. Schlieren images for run 3498 with overlaid CFD solution

\section{E. Laser Induced Incandesce (LII) and extinction Measurements}

In an effort to better quantify the level of graphite particulate in the AEDC Tunnel 9 Mach 10 flowfield, laser-induced incandescence (LII) and extinction measurements were performed. The principle of the LII technique is the detection of the thermal radiation (incandescence) from carbon particles that have been heated to near vaporization temperatures. The heating is accomplished by shaping the infrared $1064 \mathrm{~nm}$ output from a pulsed, high-energy Nd:YAG laser into a thin sheet of light and directing this light sheet across the measurement volume of interest. The extinction measurement is achieved by shaping the frequency-doubled $532 \mathrm{~nm}$ output of the same Nd:YAG laser into a similar light sheet and overlaying this sheet of light with the infrared sheet of light. The LII measurement required considerable laser energy $(400 \mathrm{~mJ} /$ pulse $)$, whereas the extinction measurement needed only around $50 \mathrm{~mJ} / \mathrm{pulse}$. This was achieved by externally controlling both the flashlamps and the Q-switch and rotating the doubling crystal so that only a small amount of the Nd:YAG energy was converted to visible light. For the Tunnel 9 application, the light sheet dimensions were approximately $40 \mathrm{~mm}$ wide by $1 \mathrm{~mm}$ thick and oriented horizontally and directed across the core flow of the tunnel.

Prior to forming the visible light sheet, the $532 \mathrm{~nm}$ beam was split into two beams, one used as a reference and the other for the extinction measurement. In order to remove elastically scattered light, which was generated from reflections from tunnel walls and windows, the reference beam was directed to the top of the Tunnel 9 test cell where it was propagated through a quartz cuvette which contained a diluted Rhodamine 610 dye/methanol solution, creating a laser-induced fluorescence signal (LIF) which was directly proportional to the beam intensity and at a wavelength substantially different from the $532 \mathrm{~nm}$ light. The reduced intensity of the extinction was quantified by reforming the beam into its original round shape and directing it to the top of the Tunnel 9 test cell from the opposite side and then through the same quartz dye cell, slightly below the reference beam, creating a second LIF image. An ICCD detector monitored the LIF images from the two beams. In order to eliminate elastically scattered (532 $\mathrm{nm}$ ) light, a $610 \mathrm{~nm}$ bandpass filter was placed between the dye cell and the ICCD detector. Figure 7 shows the dye cell and the reference and extinction beams before and during Run 3497. By comparing the ratio of the extinction beam to the reference beam to the ratio of the two beams prior to the tunnel run, it was possible, using an Abel inversion technique, to determine the relative average mass fraction of the graphite (parts per million) during the tunnel run (28). 


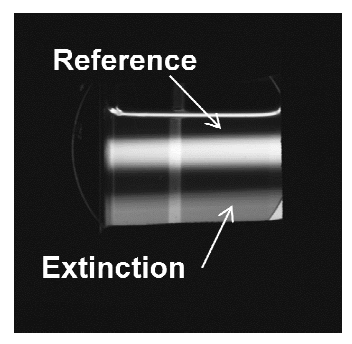

Pre-run 3497

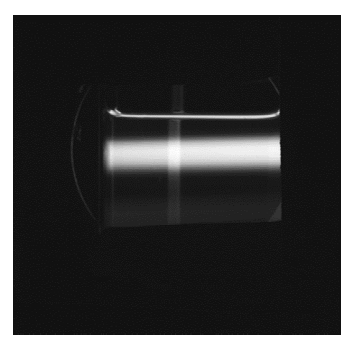

During run 3497

(85\% extinction)

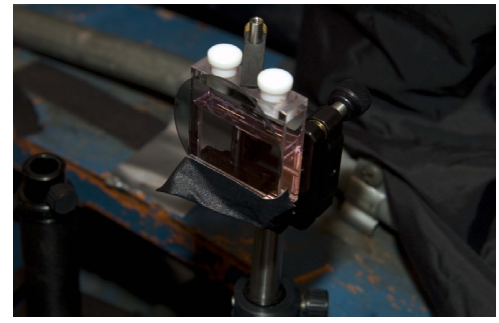

Rhodamine dye cell

\section{Figure 7. Rhodamine dye cell and Pre / during Run 3497 extinction}

In order to avoid disturbing the graphite distribution, the $1064 \mathrm{~nm}$ beam was delayed approximately $10 \mathrm{nsec}$ relative to the $532 \mathrm{~nm}$ beam. The delay was accomplished by reflecting the beam between a series of mirrors over a total distance of approximately $10 \mathrm{ft}$, or $10 \mathrm{nsec}$ (speed of light equals $0.984 \mathrm{ft} / \mathrm{nsec}$ ). The incandescence was recorded using two ICCD detectors, each having a spectral observation window defined by a short-pass filter centered at 450 $\mathrm{nm}$. In order to calibrate the system, the integration, or gate time of both cameras was set to be coincident with the laser light pulse. Subsequently, for the LII decay measurement, one camera was gated on during the laser pulse, and the other gated on slightly after the laser pulse, in order to measure the incandescence decay rate, which is an indicator of the average particle size. In essence, large particles cool more slowly than small particles. The particle size is determined by comparing the decay rate to experimental curves ${ }^{(29)}$.

\section{F. Data Acquisition and Reduction}

1. Data acquisition Systems

Two data systems were used during the test program. Data collected at lower sample rates (up to 100,000 samples per second) were acquired using the Tunnel 9 Data Acquisition and Recording Equipment (DARE) IX. Tunnel 9's HBM Genesis 5i (Gen5i) was utilized to obtain data at sample rates greater than 100,000 samples per second.

DARE IX can simultaneously acquire 16-bit data from 480 channels at user selectable sampling rates up to 10,000 samples per second and 12-bit data from 32 channels at user selectable sampling rates up to 100,000 samples per second. The input signals from all DARE channels were amplified and fed through six-pole linear phase lowpass analog filters to avoid aliasing. Data were collected at either $10,000 \mathrm{~Hz}$ or $100,000 \mathrm{~Hz}$ depending on the sensor location. All data acquired at 10,000 was analog filtered with a low-pass cutoff frequency of 2,550 Hz. The data channels that were acquired at $100,000 \mathrm{~Hz}$ were low-pass analog filtered with a cutoff frequency of $25,500 \mathrm{~Hz}$.

High-frequency dynamic-surface-pressure (PCB) and heat-flux (ALTP) data were recorded with a HBM Genesis 5i high-speed data system.. The Gen5i can acquire 16-bit data on 8 channels at sample rates of 1E6 samples per second and 15-bit data on 12 channels at sample rates of 25E6 samples per second.

\section{Heat Transfer}

Heat transfer was acquired using Type-E Chromel-Constantan coaxial thermocouples. The millivolt output of each thermocouple is converted to absolute temperature based on the initial reference temperature measured by two of the thermocouples on the model that were recorded using electronic cold junction compensators. The model was assumed to be isothermal at the beginning of the run. The absolute temperature is determined from the NIST ITS-90 coefficients for a Type-E thermocouple.

The heating rate for the coaxial thermocouples were computed from the full temperature history of each gage using an explicit finite difference solution to the unsteady, one dimensional heat conduction equation for a homogeneous planar slab of finite thickness ${ }^{(30)}$. The thermocouples are mounted into the model directly which was fabricated from 15-5 stainless steel with a heat treat of H900. Temperature-dependent thermal properties were used throughout the calculation based on results documented in ${ }^{(31)}$. The local geometry of the model surface required planar, cylindrical or spherical coordinates to account for the wall thickness and surface curvature of each gage location. The backface temperature rise was negligible for the model; thus a zero heat flux backface boundary condition was assumed. The measured temperature at the surface provided the remaining boundary condition needed to compute the temperature distribution through the slab as a function of time. The heat transfer rate was then computed from the temperature gradient at the surface and the thermal conductivity of the material.

\section{G. Experimental Uncertainties}


Uncertainties in material properties used to compute the mean heat transfer from the coaxial temperature measurements are estimated at $\pm 10 \%$. The uncertainties due to variations in free stream conditions, model angle of attack, instrumentation precision, etc. is approximately $\pm 6 \%$. The root-mean-square experimental uncertainty on the mean heat transfer measurements is therefore $\pm 12 \%$. A more extensive discussion about the uncertainties on the heat transfer measurements is found in ${ }^{(2)}$ and ${ }^{(31)}$. The paper will present a discussion about the uncertainties on the pressure and heat transfer spectra.

\section{Computational Method}

Using the DPLR CFD code ${ }^{(32)}$, axisymmetric laminar Navier Stokes computations were performed on the 7-inch and 1.25-inch CEV geometries at the various test conditions. The heat transfer augmentation was obtained from the difference between the experimental and computed heat transfer. Figure 6 which presents the CFD Mach number field and streamlines superimposed on a Schlieren image displays good agreement in the shock shape and standoff distance. More detail about the CFD solutions will be given in the paper.

\section{Results and Analysis}

\section{A. Mean Heat Transfer and Heating Augmentation}

The heat transfer augmentation is assessed by comparing the heat transfer measurements to laminar CFD solutions for the 7-inch and 1.25- inch CEV models. The comparison for the various Reynolds numbers is shown in Figure 8 a) and b) for the 7-inch and 1.25-inch CEV respectively where the laminar heat transfer correlation $\left(\operatorname{StRe}_{D}{ }^{0.5}\right)$ is plotted as a function of $(\mathrm{z} / \mathrm{R})$ over the centerline. The laminar correlation collapses the CFD solutions to within a few percent. On the 7-inch CEV, apart from the 4E6/ft condition which is out of character, the discrepancy between the measurement and computation increases with Reynolds number. At the lower Reynolds number, the bulk of the deviation is localized near the stagnation point $(-0.25<z / R<0.25)$ whereas at the higher Reynolds number a significant deviation is found over the whole stagnation region. The heating augmentation on the 7-inch CEV computed as $\left(q_{E X P}-q_{C F D}\right) / q_{C F D}$ is plotted in Figure 9 for the range of Reynolds numbers. The augmentation at the stagnation point varies from approximately $10 \%$ at $R e=0.5 \mathrm{E} 6 / \mathrm{ft}$ to $40 \%$ at $R e=15 \mathrm{E} 6 / \mathrm{ft}$. The scatter among the two $R e=15 E 6 / f t$ runs is significant as it varies between 10 to $20 \%$.

Data on the 1.25-inch CEV probe shows excellent agreement between the CFD and the measurement at all Reynolds number as seen in Figure 10 and 8 b). Figure 11 shows that the model position has a negligible effect as the heat transfer data for the model mounted on centerline agrees very well with the model mounted off the tunnel centerline.

Figure 12 illustrates the effect of the model size by plotting the 7-inch and 1.25-inch CEV heat transfer data at similar $R e_{D}$. Clearly, the stagnation point heating augmentation only occurs on the larger CEV model and the augmentation increases with Reynolds number.

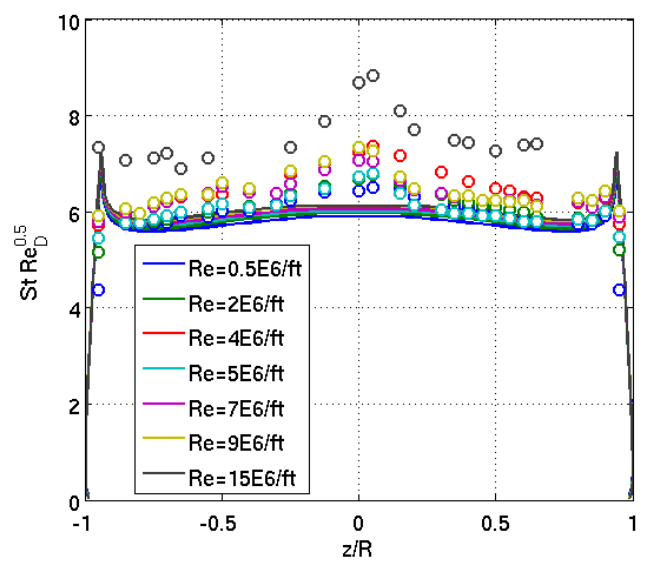

(a) 7-inch CEV

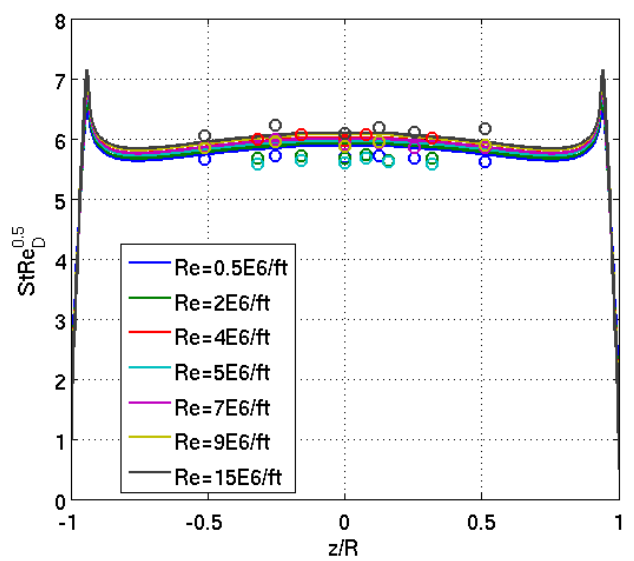

(b) 1.25-inch CEV

Figure 8. Comparison between experimental and computed laminar heat transfer correlation on centerline

10

American Institute of Aeronautics and Astronautics 

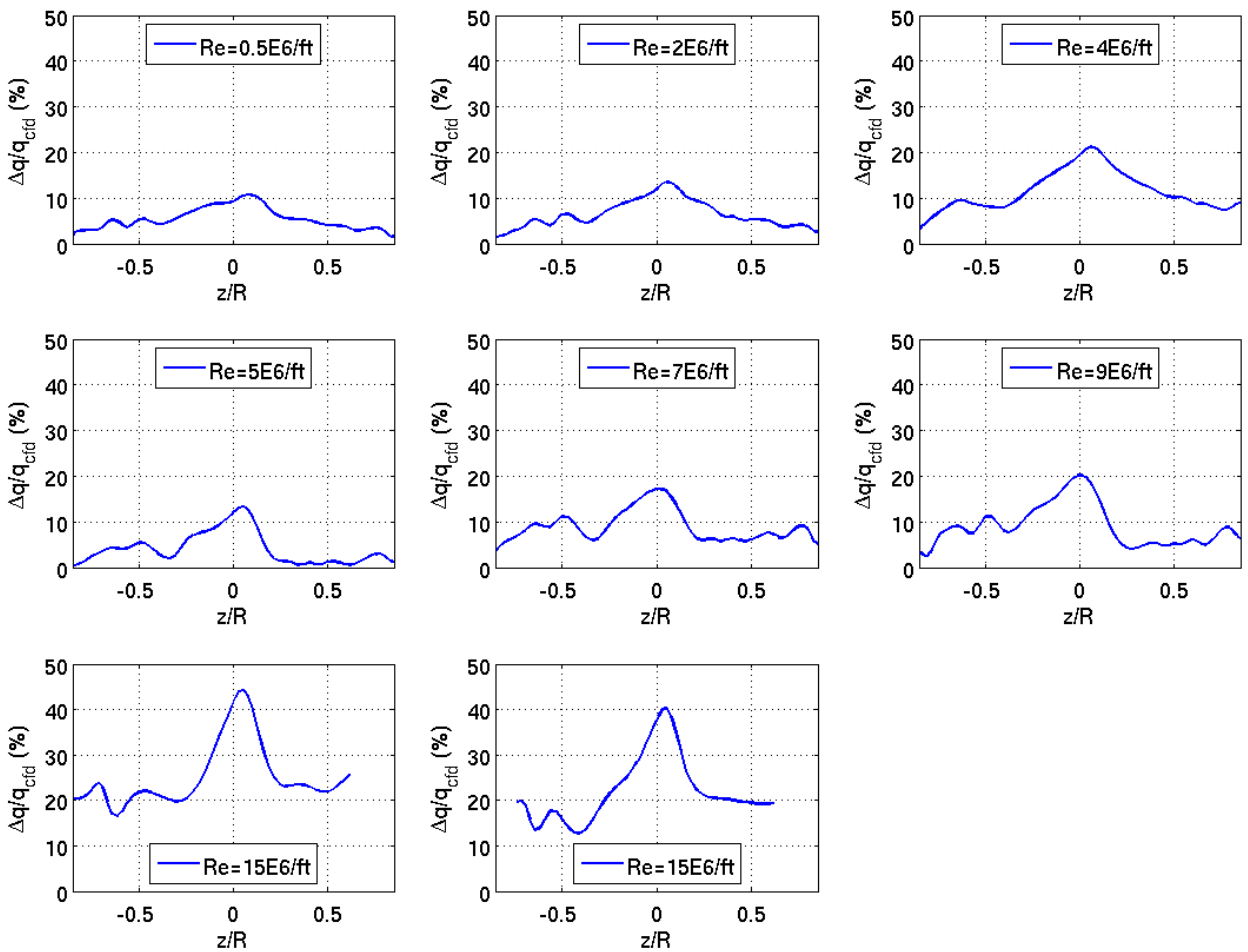

Figure 9. Heating augmentation on centerline computed as $\left(q_{E X P}-q_{C F D}\right) / q_{C F D} \%$ 

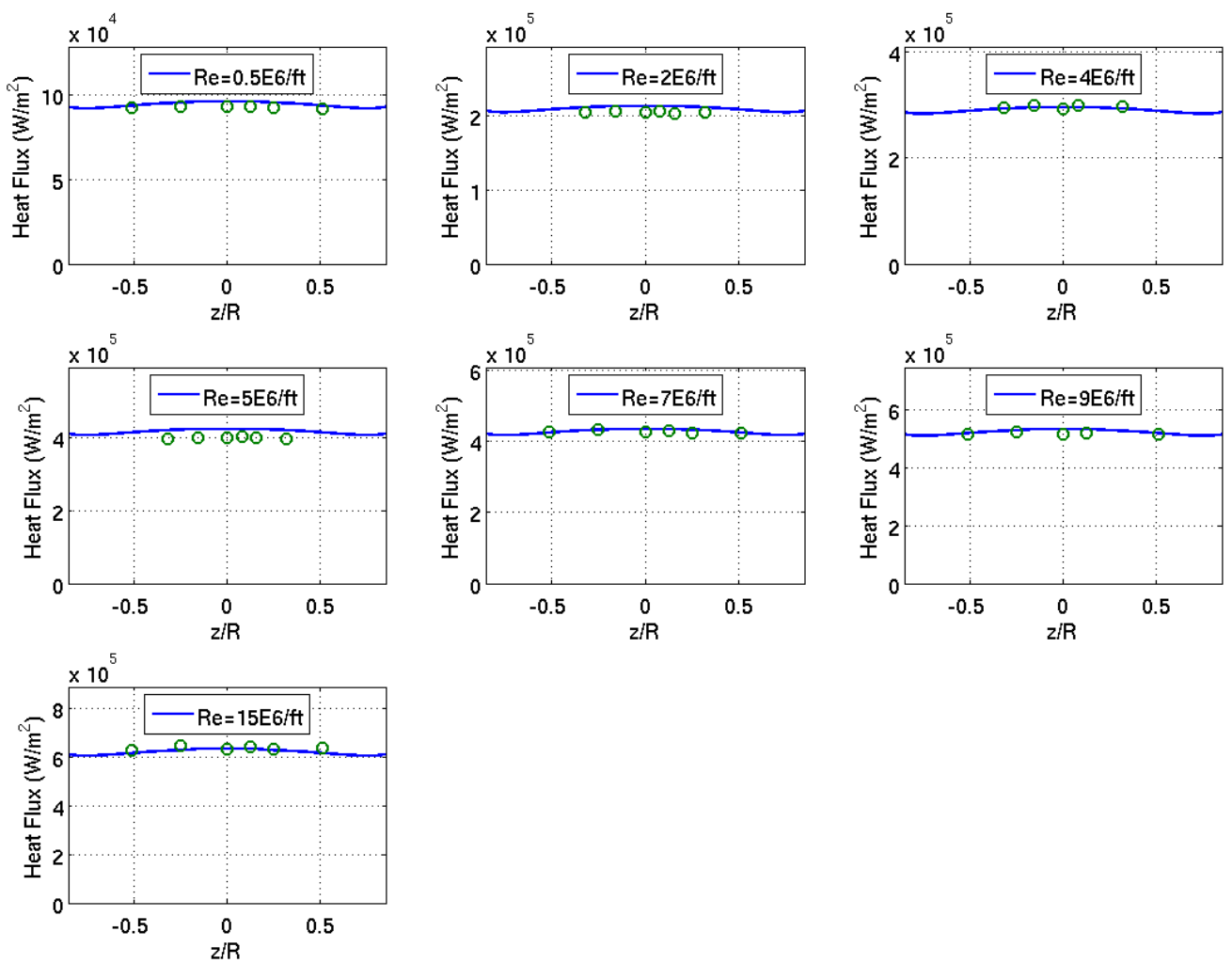

Figure 10. Comparison between computed and measured centerline heat flux on 1.25-inch CEV

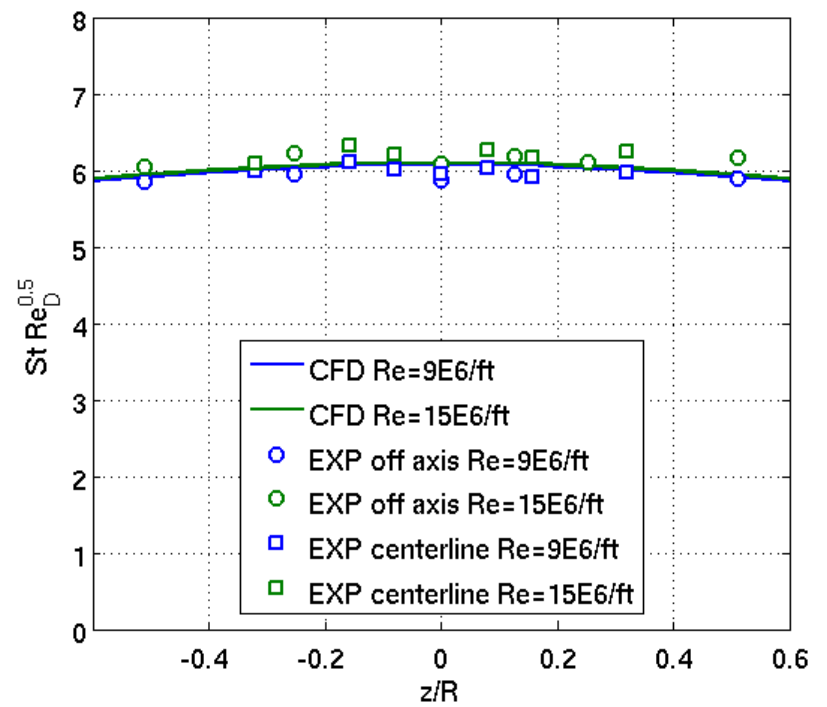

Figure 11. Effect of model position on heat transfer 


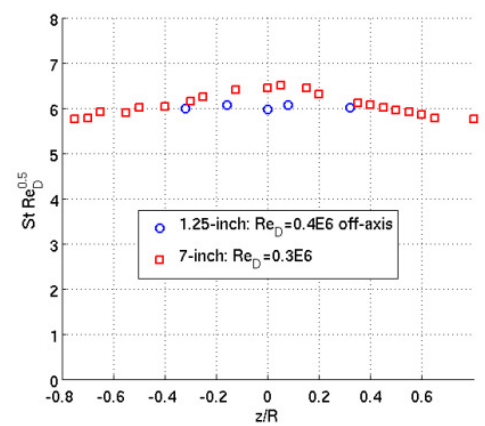

(a) $0.3 \mathrm{E} 6<R e_{D}<0.4 \mathrm{E} 6$

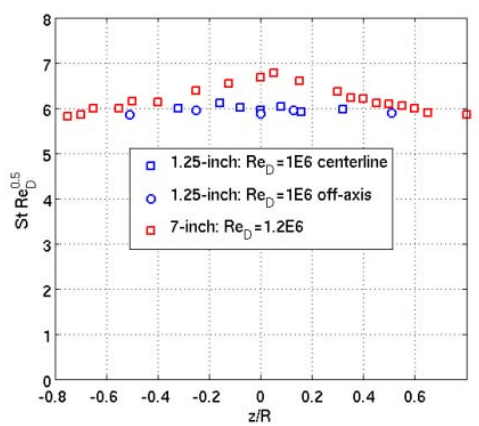

(b) $1 \mathrm{E} 6<\mathrm{Re}_{\mathrm{D}}<1.2 \mathrm{E} 6$

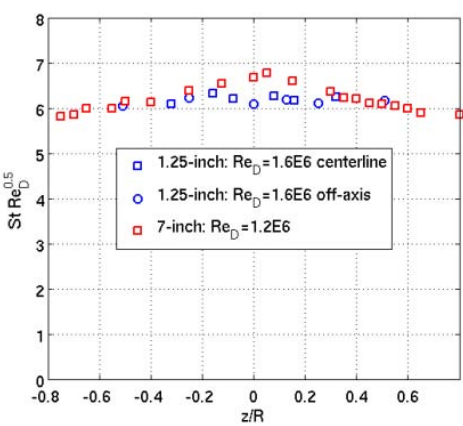

(c) $1.2 \mathrm{E} 6<R e_{D}<1.6 \mathrm{E} 6$

\section{Figure 12. Comparison of laminar heat transfer correlation on centerline between 1.25- inch and 7-inch CEV at similar $\operatorname{Re}_{D}$}

\section{B. Characterization of Graphite Particulates}

Fine graphite particulates are expected to be present in the test gas because of the graphite heater It has been hypothesized that this particulate could have an effect on the stagnation region heat transfer due to the added energy from impacts and added vorticity in the wake and the particulate/shock layer interaction. The mass fraction of graphite particulates obtained from the extinction measurements described in section III E is plotted as a function of the Reynolds number in Figure 13. The mass fraction increases with free stream Reynolds numbers up to $R e=4 \mathrm{E} 6 / \mathrm{ft}$ after which it remains approximately constant at approximately 0.4 part per million. We believe that the energy addition from such a small mass fraction has a negligible effect on heat transfer. This assertion will be verified more carefully in the paper.

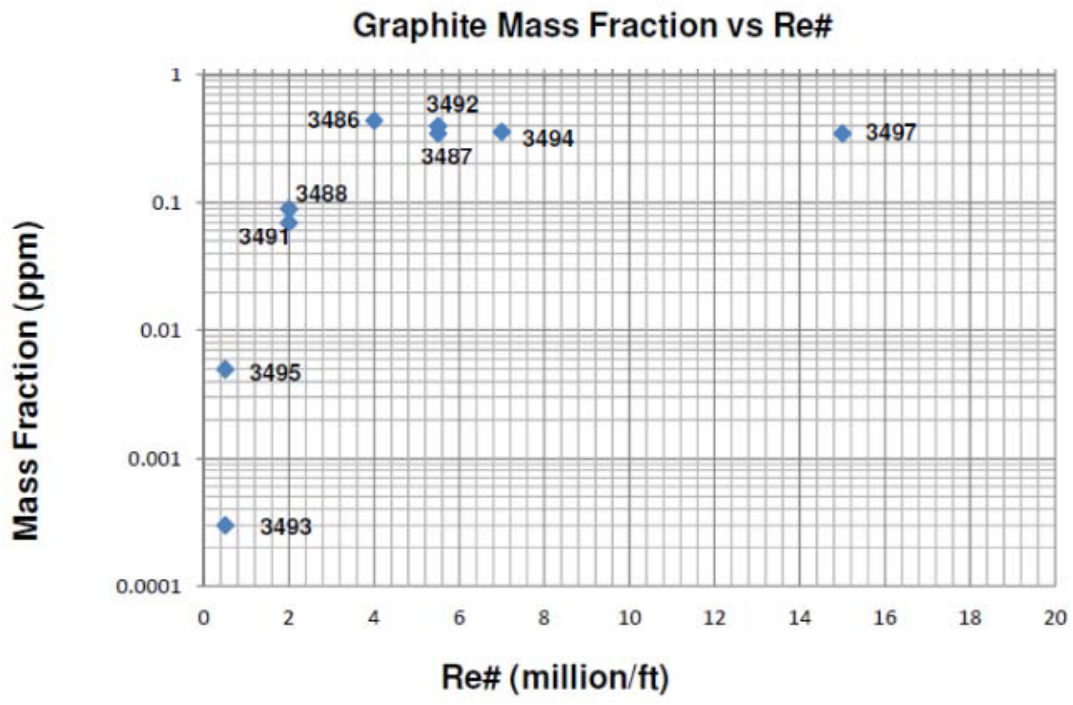

Figure 13. Mass fraction of graphite as a function of free stream Reynolds number

A low mass fraction of particulates might still have an influence on heat transfer if the size of the particulates is large enough to inject a significant amount of vorticity in the stagnation region (13). Average particulate size were obtained from LII measurements for runs 3492 (at $R e=5.5$ millions/ft) and 3497 (at $R e=$ $15 \mathrm{E} 6 / \mathrm{ft})$. For both cases, the average particle size is below $10 \mathrm{~nm}(0.01$ micron) in diameter which corresponds to the resolution of the measurement and much smaller than the ones investigated by Holden ${ }^{(13)}$. Since the size of the particulates is much smaller than the size of both the 7 and 1.25-inch CEV models, the effect of particulates on heating is expected to be the similar on both models at the same $R e_{D}$. The fact that augmented heating is absent on the 1.25-inch CEV for values of $R e_{D}$ where it is present on the 7-inch CEV seems to rule out the graphite particulates as a significant contributor to the stagnation point heating augmentation. 


\section{Effect of Larger Particle Impacts}

High resolution Schlieren movies taken during a previous experimental program on the Orion CEV capsule ${ }^{(2)}$ have shown occasional large disturbances to the shock layer. Such disturbances are attributed to impacts from larger particles possibly fragments of Delrin ablators used as flow restrictors during the tunnel startup. During the previous program ${ }^{(3)}$, the frame rate was not large enough to resolve the details on an impact and the possibility of missing a fraction of the impacts was brought up.

To address the previous limitations, high speed Schlieren movies at a frame rate of $15 \mathrm{kHz}$ were obtained during this program. Figure 14 displays a series of 20 Schlieren frames $(\sim 1.33 \mathrm{msec})$ illustrating the evolution of the disturbances created by a large particle. The particle which initially travels near the free stream velocity collides with the model wall. Subsequently, the reflected particle and/or fragments ejected from the model surface move ahead of the bow shock generating a shock around the particle (or the fragments) which interacts with bow shock as seen in frame 2 to 16 . The shock/shock interaction induces a shear layer which penetrates the stagnation region and dramatically increases heat transfer. Aerodynamic drag decreases the particle velocity up to a point where it starts moving back towards the body and eventually reenters the shock layer. Due to its lower momentum compared to the initial collision, the particle remains in the shock layer where it is radially pushed out of the stagnation region by the flow. As seen in Figure 14, it takes between 1 and 2 milliseconds for the flow to return to its undisturbed state following the initial collision.

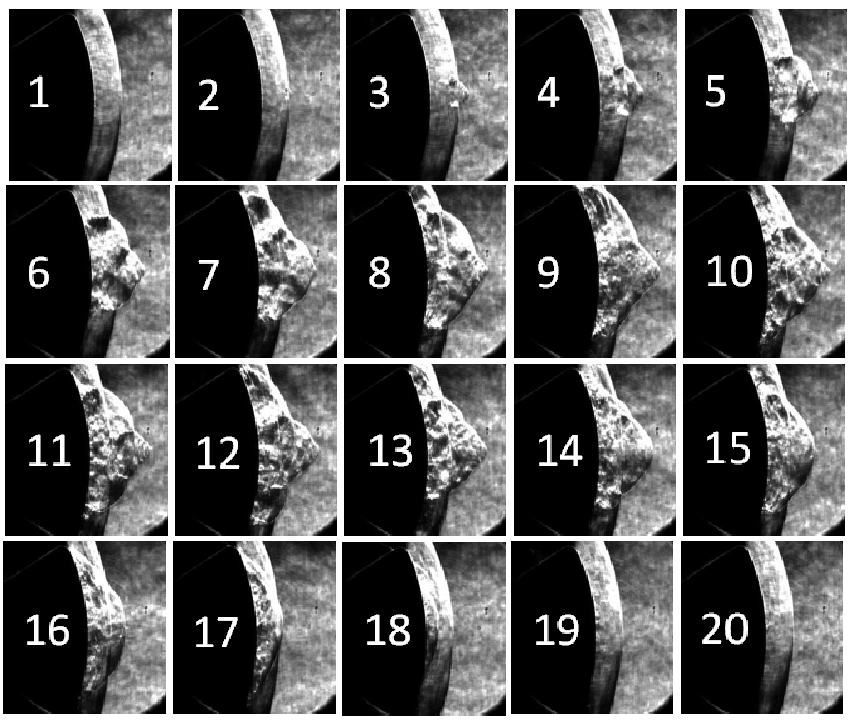

Figure 14: Large impact on 7-inch CEV recorded at $15 \mathrm{kHz}$

During the initial CEV test program ${ }^{(2)}$, the thermocouple sampling rate was too low to resolve the effect large impacts on heat transfer. Some suspected that the increase in average heat transfer was due to the temporal averaging of the intermittent heat transfer enhancements from several large collisions. It was also suggested that the increased heating persisted for a significant time following an impact which would further increase the measured heat transfer.

For the current program, several thermocouples were sampled at $100 \mathrm{kHz}$. In addition, several ALTP sensors were used to obtain time resolved heat transfer data. Figure 15 presents the effect of large impacts on the heat flux obtained with ALTP sensors. As seen in the high speed movies, the heat transfer recovers its pre-impact value in less than 5 milliseconds and no significant difference is found in the pre and post-impact heat transfer rates. In order for the large impacts to exert a significant effect on the measured heat transfer, the occurrence (or probability of impacts) needs to be sufficient. Since the impacts always exert an increase above the undisturbed heat transfer, the probability of impact can be computed by looking at the positive tail of the probability density function (PDF) of the heat transfer fluctuations. The PDF for run 3497 (at $R e=14.9 E 6$ ) is shown in Figure 16 a) along with a normalized Gaussian PDF. A large deviation from the Gaussian PDF is found. However, the probability of the heat flux being higher than 5 standard deviations remains small at less than $1 \%$. The effect of large impacts on heat transfer is quantified by removing the elevated heat flux data point from the averaging. Here, the threshold for data removal set to +8 standard deviations. The PDF for run 3497 with removed impacts is shown in Figure $16 \mathrm{~b}$ ) where it now closely agrees with the normalized Gaussian PDF. The computed increase in heat transfer due to large particulates is 
between 0.2 and $4.5 \%$. The effect increases with Reynolds number, but only represents at most $10 \%$ of the measured heating augmentation. Large impacts are therefore not the main contributor for the measured heating augmentation. The paper will present a table with the effect of the large particle impacts on heat transfer for each condition.

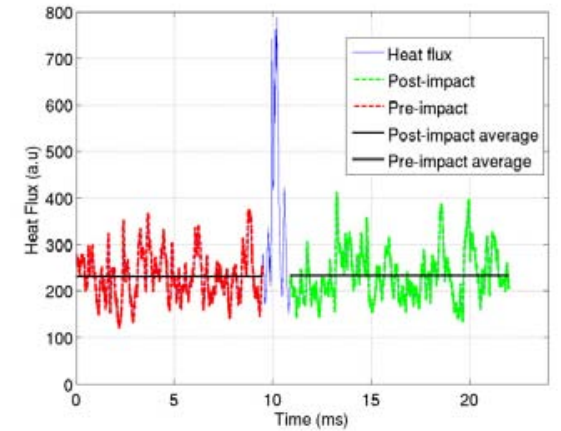

(a) Run 3492

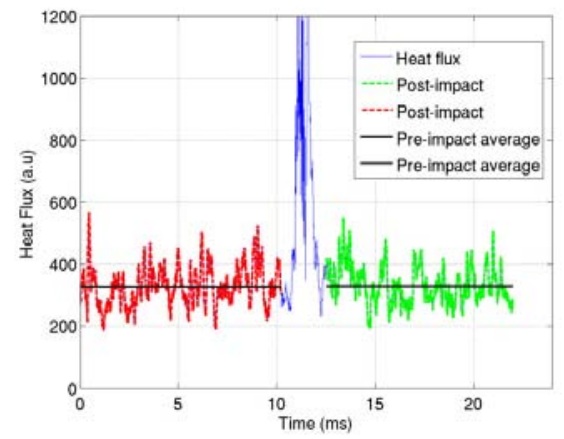

(c) Run 3496

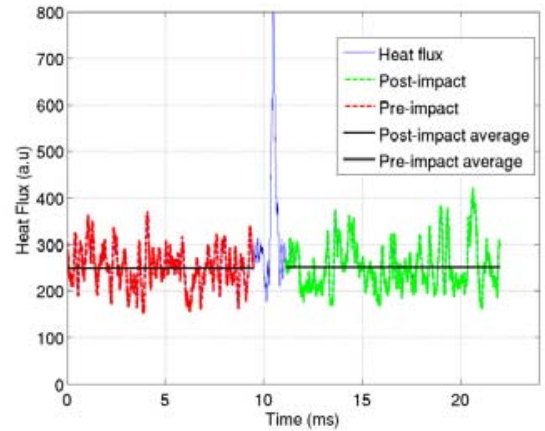

(b) Run 3494

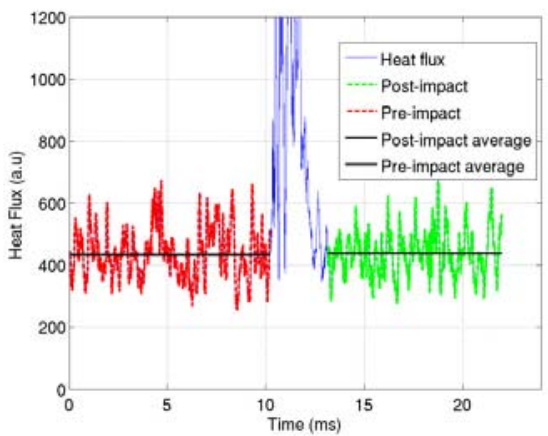

(d) Run 3497

Figure 15. Effect of large particle impact on heat transfer

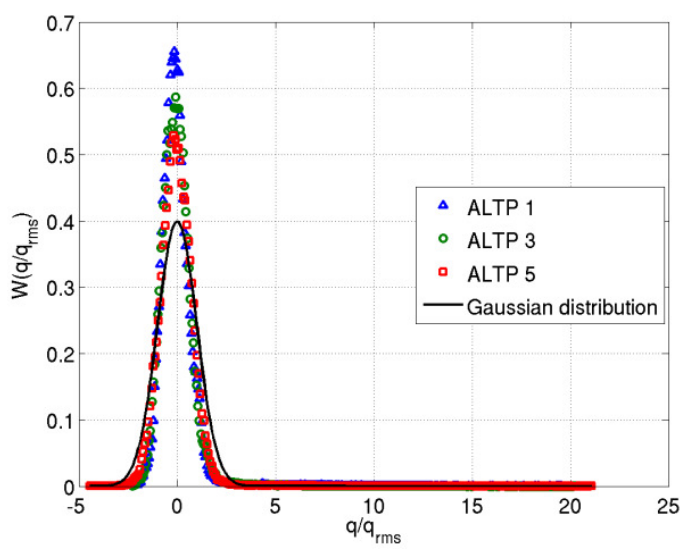

(a) PDF with impact

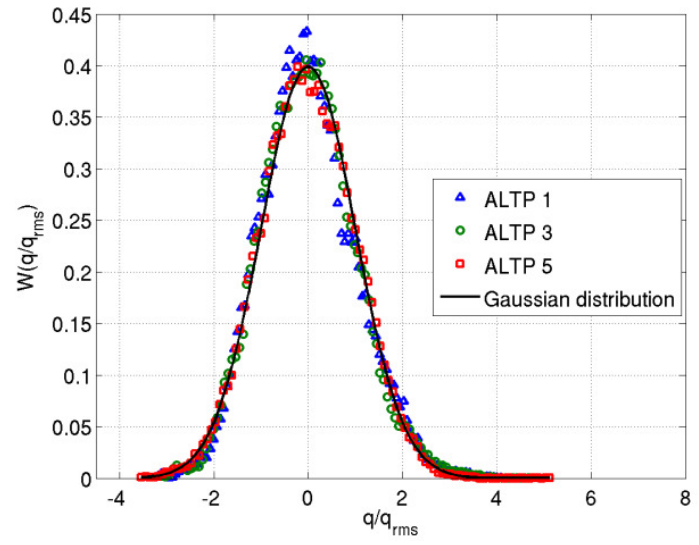

(b) PDF without impact

Figure 16. Effect of large impacts on probability density function (PDF) for run 3497

\section{Characterization of Pressure and Heat Transfer Fluctuations}


Properties of the pressure and heat transfer fluctuations are investigated to understand the effect of model size on the stagnation point heat transfer fluctuations. The effect of large particle impacts was removed from the fluctuation data by removing samples in regions where the fluctuation exceeded 8 standard deviations (see section V C)

\section{Heat Transfer and Pressure RMS}

Figure 17 presents the normalized RMS heat flux and pressure fluctuations (in percent) as a function of the radial distance from the stagnation point $(r / R)$ at the various Reynolds number. Both the heat flux and pressure fluctuations RMS decrease with increasing Reynolds numbers. This is consistent the tunnel noise decreasing with Reynolds number as reported in ${ }^{(27)}$. A decrease in the heat flux RMS with radial distance is observed as opposed to the pressure RMS which is either approximately constant or increasing with distance. The heat flux RMS is always significantly larger than the pressure with the ratio of heat flux to pressure RMS varying between 2 and 20 where the greatest ratios are near the stagnation point.
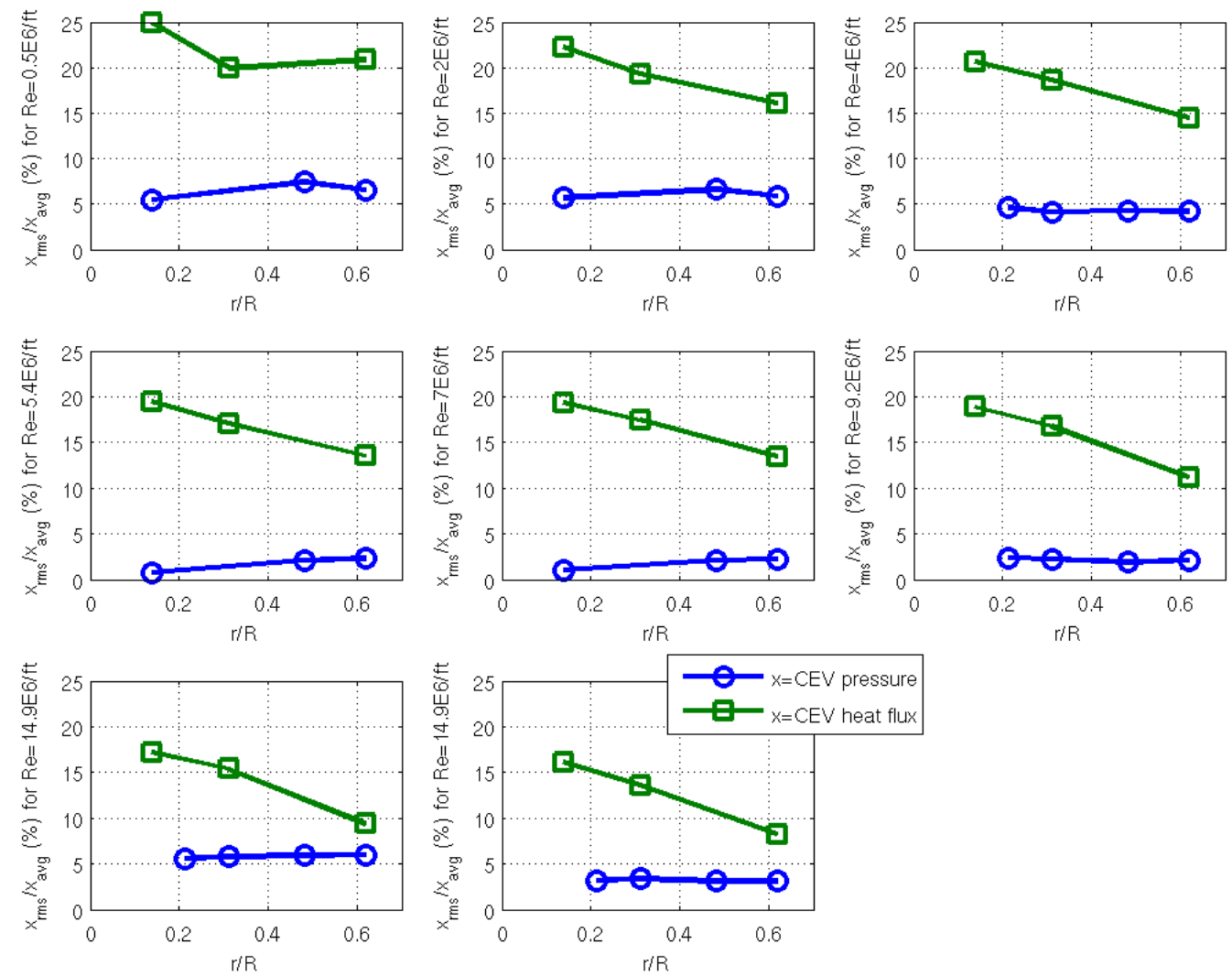

Figure 17. Pressure and Heat Transfer RMS as a function of position

\section{Pressure and Heat Transfer and Power Spectral Density}

The Power Spectral Density (PSD) of the pressure fluctuations (normalized by the mean pressure) is plotted in Figure 18 a) and b) for the Kulite noise probe and 7-inch CEV Kulite PHS4 sensor located at $r / R=0.24$. The same scale is used in both figures to facilitate comparisons. In both cases, the PSD is essentially flat for frequencies up to $2 \mathrm{kHz}$ excluding very low frequency bumps which might be due to the limited spectral resolution. Striking differences exist between both PSDs as a much greater fraction of the energy is present at low frequencies (below 3 $\mathrm{kHz}$ ) for the 7-inch CEV. For instance, at the Re=14.9E6/ft condition, the ratio of energy in the $0-3 \mathrm{kHz}$ band to than in the $3-30 \mathrm{kHz}$ band is equal to 2.5 for the 7 -inch CEV compared to 0.8 for the noise probe. The attenuation at high frequency displays a $f^{-1}$ dependence, for the noise probe, compared to a much greater attenuation on the 7-inch CEV where a $f^{-11 / 3}$ dependence is observed. The increased attenuation a high frequency could be indicative of the pilling-up of eddies near the stagnation points, since a partial cancellation of the fluctuations induced by the small eddies is known to occur ${ }^{(33)}$. Inside the flow, near the stagnation point, rapid distortion theory predicts a $f^{-11 / 3}$ dependence ${ }^{(33)}$ whereas on the surface, further attenuation occurs and a $f^{-16 / 3}$ dependence is predicted ${ }^{(33)}$ (34). Pressure measurements at the stagnation point of a two dimensional square prism submitted to free stream 
turbulence was performed by Kawai et al. ${ }^{(35)}$ in low speed flow. A $f^{-3}$ dependence was measured which is similar to what was observed on the CEV.

A significant Reynolds number effect is observed at high frequency as the PSD becomes flat. This is best seen at $R e=0.5 \mathrm{E} 6 / \mathrm{ft}$ where the PSD is flat at frequencies above $10 \mathrm{kHz}$. As seen in Figure $18 \mathrm{~b}$ ), the electrical noise obtained before the run is much lower than the signal such that the flat region is not because of electrical noise. The flat portion at high frequency is also present in the free stream noise as best seen at $R e=2 \mathrm{E} 6 / \mathrm{ft}$. (noting that noise was not measured at $R e=0.5 E 6 / f t$ ). The extent of the $f^{-11 / 3}$ region shrinks down with decreasing Reynolds numbers. This Reynolds number effect is not well understood.

The heat flux fluctuations PSD is found in Figure 19 a) and b) for the 0.875 -inch flat face probe at $\mathrm{Re}=2 \mathrm{E} 6 / \mathrm{ft}$ and ALTP sensor 3 located at $r / R=0.31$ on 7 -inch CEV. The same scale is used in both figures to help comparison. As for the pressure PSD, the CEV model has more low frequency fluctuations. Here, the difference is more significant than for pressure as about ten times more energy is present at low frequency on the 7 -inch CEV. On the ALTP probes, a $f^{-7 / 3}$ dependence is found at frequencies above $10 \mathrm{kHz}$. On the 7 -inch CEV, the attenuation starts at lower frequencies and the slope, equal to $-9 / 3$, is steeper such that the energy content is similar at $100 \mathrm{kHz}$. The low frequency bumps present in the pressure PSD are not found for both the flat face probe for 7-inch CEV heat flux PSD. As seen in the Pressure PSD, the extent of the constant slope region shrinks down with decreasing Reynolds number. Unlike for pressure, the PSD doesn't become perfectly flat, but at significant change in slope is observed. The change in slope moves to higher frequencies as the Reynolds number increases.

The main finding from the spectral analysis is that the 7-inch CEV model significantly modifies the incoming pressure and heat flux disturbances as the low frequency content and high frequency attenuation both increase on the larger model.

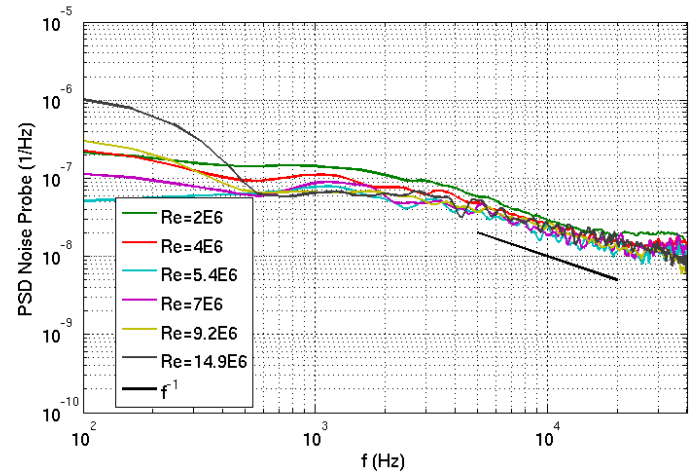

(a) Noise Probe (Kulite)

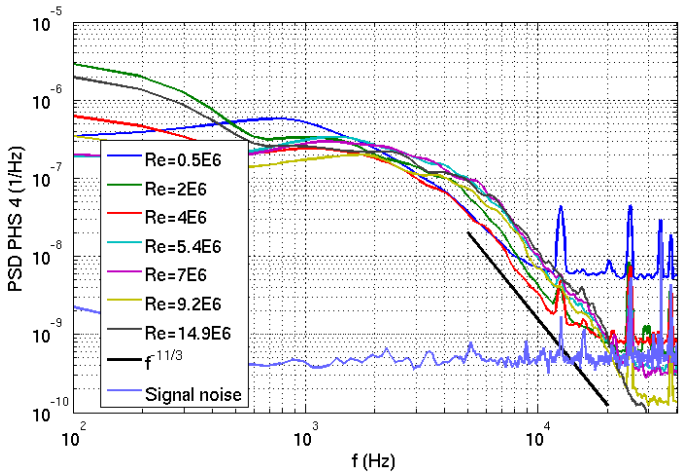

(b) Kulite Sensor 4 (PHS 4)

Figure 18. Pressure PSD for noise probe and wall pressure sensor (PHS 4) on 7-inch CEV

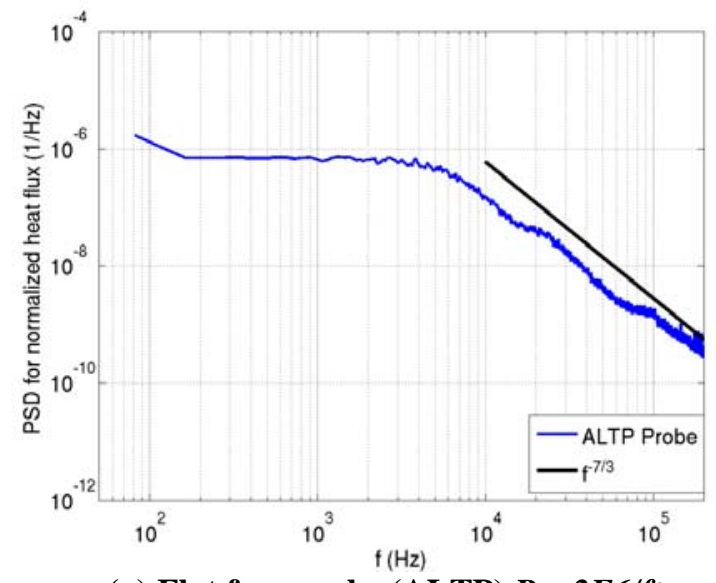

(a) Flat face probe (ALTP) $R e=2 E 6 / f t$

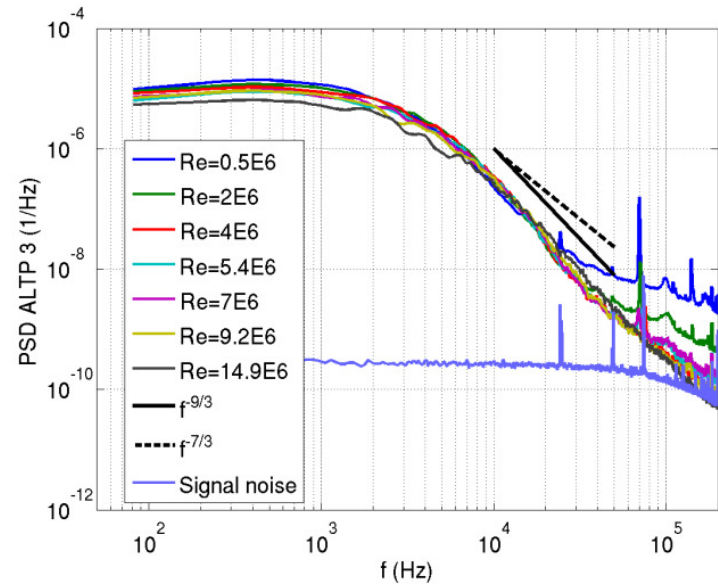

(b) ALTP 3

Figure 19. Heat flux PSD for flat face probe and surface ALTP 3 on 7-Inch CEV 


\section{E. Convective Velocity and Length Scales of Fluctuations}

To better understand the nature of the heat transfer fluctuations on the 7-inch and 1.25-inch CEV models, spacetime correlations of surface heat transfer fluctuations are performed using pairs of thermocouples. Figure 20 a) presents sample correlation coefficients at $R e=4 E 6 / f t$ for the sensor pairs designated in Figure $20 \mathrm{~b}$ ). With the exception of pair $t 60-t 28$, the sensor pairs are chosen along streamlines. For pair $t 73-t 53$ the assumption of being along a streamline is not exact Sensors located along streamlines (or approximate streamlines) display a positive correlation coefficient and a positive time delay between the downstream and upstream sensors. In contrast, the $t 60$ t28 pair which is not located along a streamline is uncorrelated. Therefore, fluctuations appear to be traveling along streamlines. This lack of correlation was also found for other sensor pairs not located along streamlines which are not shown in Figure 20.

By investigating the convective velocity, additional knowledge is gained about the nature of the fluctuations. The convective velocity is computed by dividing the streamwise distance by the time delay corresponding to the position of the maximum correlation coefficient. We assume that the computed velocity is that at the midpoint between the pair of sensors. The convection velocity as a function of the streamwise distance at the different Reynolds number is shown in Figure 21. For comparison, the boundary layer edge velocity obtained with CFD is also shown. The runto-run variation in edge velocity was found to be small such that only one CFD solution is shown. Despite some scatter, the convective velocity increases linearly with distance from the stagnation point and agrees quite well with the CFD solution. A linear fit of the experimental data is also shown. The analysis shows that the disturbances are approximately convecting at the boundary layer edge velocity. This finding implies that they are due to vortical disturbances (vorticity mode) and/or temperature spots (entropy mode). It rules out acoustic disturbances as a significant contributor since they travel at velocities $u \pm a$ in every possible direction because the flow is subsonic over the stagnation region.

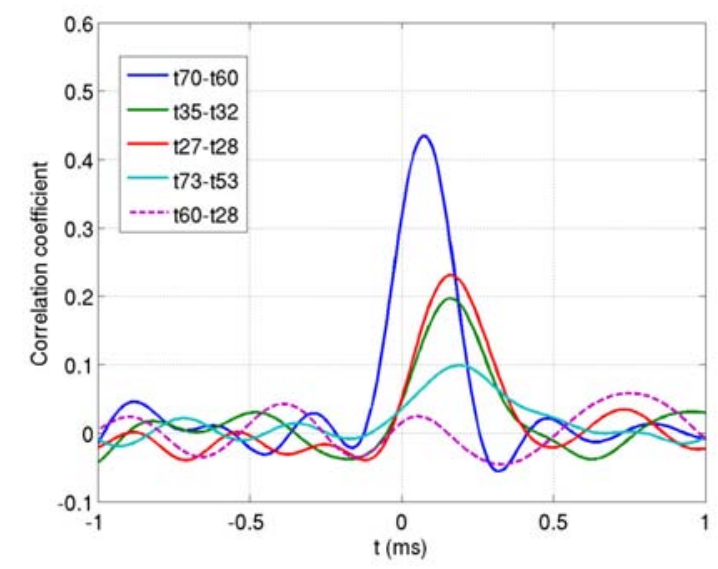

(a) Correlation coefficients for thermocouple pairs

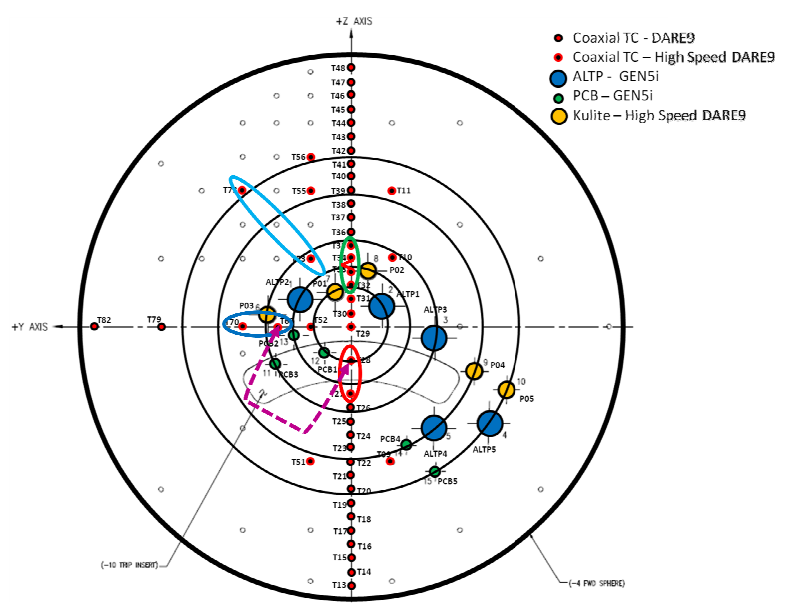

(b) Thermocouple pairs

Figure 20. Correlation coefficients from thermocouple pairs for $\operatorname{Re}=4 E 6$ on 7 -inch $\mathrm{CEV}$ 


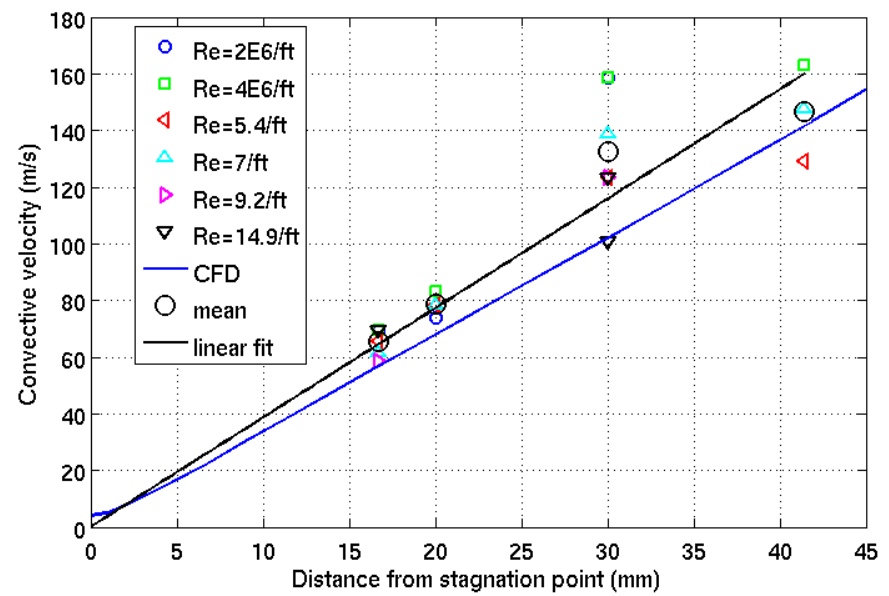

Figure 21. Convective velocity as a function of distance for 7-inch CEV

The same space-time correlation analysis is performed for the 1.25-inch CEV model. The results and thermocouple pairs are shown in Figure 22 a) and b) respectively. The difference with respect to the 7-inch CEV is striking as gages located on opposite side of the model are correlated on the 1.25-inch CEV model (ta01-ta07 and ta02-ta05 pairs). The correlation of opposing sensors implies that the disturbances are larger than the thermocouple spacing which is equal to 0.64 inch for pair ta01-ta07 and 0.24 inch for pair ta02-ta05. The disturbances are therefore large with respect to the model size which is also corroborated by the zero or near zero time delay between the sensors indicating that both sensors sense the disturbances at the same time.

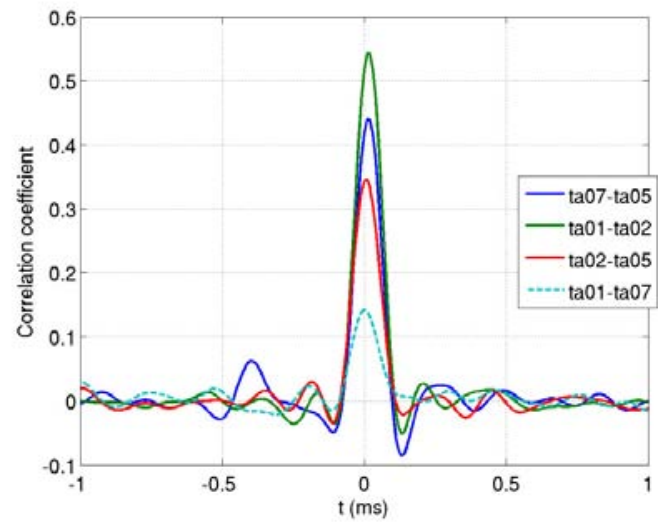

(a) Correlation coefficients for thermocouple pairs

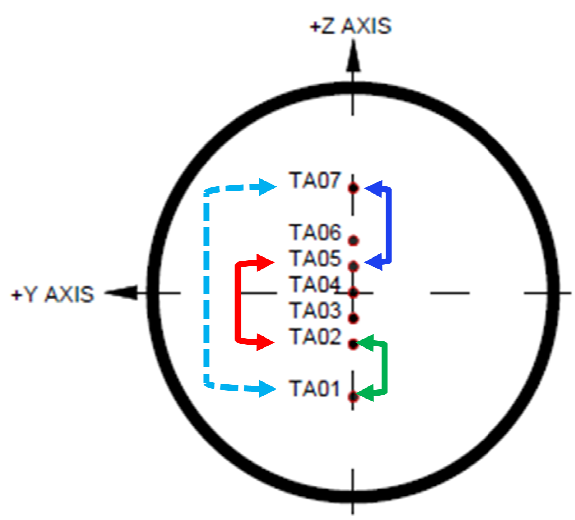

(b) Thermocouple pairs

\section{Figure 22. Correlation coefficients from thermocouple pairs for $R e=4 E 6$ on 1.25 -inch $\mathrm{CEV}$}

The integral time scale of the heat flux disturbances is computed by integrating the autocorrelation function of the heat flux fluctuations for both the ALTPs and coaxial thermocouples. Assuming that the disturbances are convected at the edge velocity, the integral length scale can be computed as the product of the integral time scale and the convective velocity over the sensor. The integral length scale as a function of distance from the stagnation point is plotted in Figure 23 for both the ALTPs and coaxial thermocouples where the dimensions are normalized by the heat shield radius (3.5-inch). The integral length scale increases linearly with distance from the stagnation point. It is of the order 0.2 -inch at the first measurement station located near the stagnation point to 0.6-inch at $r / R \approx 0.6$.

This increase in scale could be an indication that vortical structures (vortex filament) are getting stretched in the streamwise direction with increasing distance from the stagnation point. This stretching predicted by RTD can be intuitively understood by observing that the head of the vortex filament travels faster than its tail thus increasing its length in the streamwise direction. The ALTP measurements expose a decrease in the integral scale with increasing 
Reynolds number an effect not distinguished in the coaxial thermocouple measurements which is most likely due to their limited frequency response.

The scale of the disturbances in the free stream can be estimated with the 0.875-inch ALTP face probe. For run 3491 at $R e=2 E 6 / f t$, the integral time scale is about 34 microseconds for a frequency of about $30 \mathrm{kHz}$ which is larger than the frequency response of the coaxial thermocouples. We are therefore restricted to ALTP measurements. When the probe scale is very small with respect to the scale of the incoming disturbances, we can assume that the disturbances travel at the free stream velocity over the probe such that the integral length scale is simply the product of the integral time scale and free stream velocity. Since the probe size is similar to that of the incoming disturbances, it is difficult to obtain an accurate scale measurement because the disturbances travel at the velocities found behind the shock layer for a fraction of the time. An integral length scale of 1.9 inch is obtained by simply multiplying the integral time scale by the free steam velocity. The measurement can be crudely corrected by assuming that the disturbance travels a distance corresponding to the shock standoff distance at the mean velocity behind the shock layer which is equal to about one tenth of the free stream velocity. Estimating the standoff distance to be $0.1 \mathrm{inch}$ for the flat face probe leads to an integral length scale of about $0.9 \mathrm{inch}$. This range of scales explains why thermocouples on opposite side of the 1.25-inch CEV are positively correlated. It is also interesting to notice that the integral length scale computed on the flat face probe is significantly larger than that found on the 7-inch CEV. This difference could indicate that the disturbances are altered by the bow shock from vortex breakdown and/or bulk compression. Another possibility is that the vortical disturbances are actually generated from the interaction of acoustic and/or entropy disturbances with the bow shock.

Future measurement of the disturbance scale sizes in the free stream should be performed with smaller probes to improve the accuracy. Using an array of small probes separated in the transverse direction would also be useful to evaluate the disturbance scale sizes with space-time correlations. The streamwise and transverse scales could be obtained by using both the autocorrelations and space-time correlations.

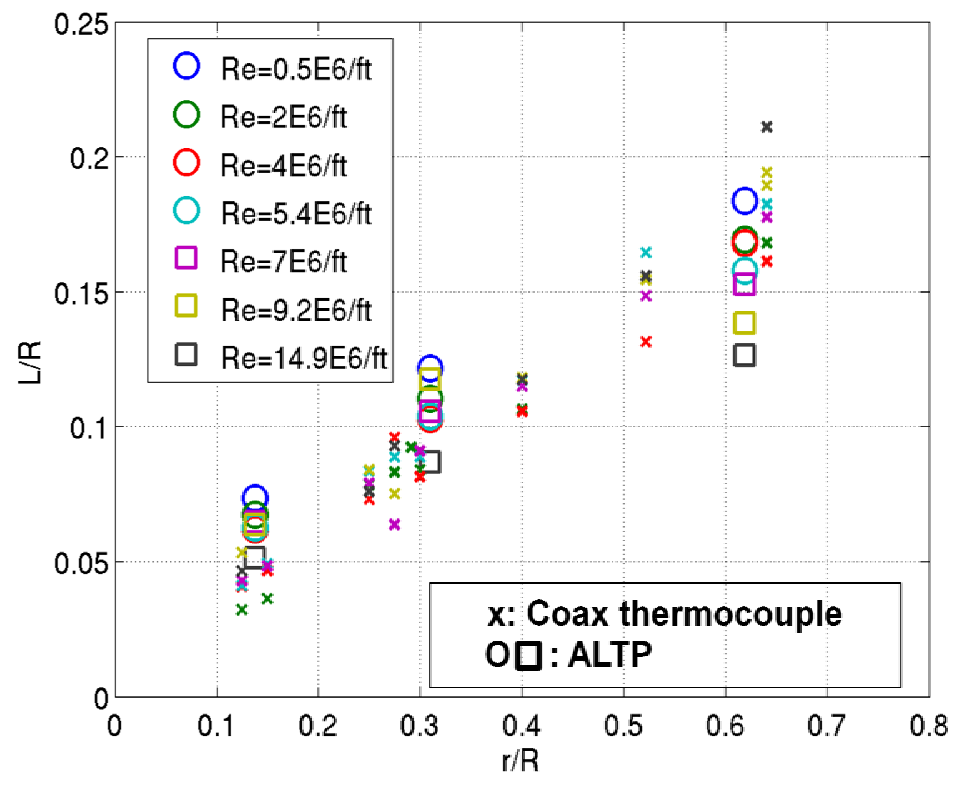

\section{Figure 23. Normalized integral length scale as a function of distance for 7-inch CEV}

\section{F. Free Stream Vorticity as the Main Mechanism for Heat Transfer Augmentation}

The increased energy at low frequency and greater attenuation at high frequency on the 7-inch CEV compared with the smaller probes is an indication that the disturbance field is modified by the large 7-inch CEV model. Moreover, the convection of the heat transfer fluctuations at the boundary layer edge velocity and the increase in the longitudinal length scale with distance from the stagnation point are indicative of the stretching and piling-up of vorticity as predicted by RDT. Therefore, the experimental evidence points towards the amplification of free stream vorticity as the dominant mechanism for the heat transfer augmentation on the 7-inch CEV model. 
As previously mentioned in Section II, the vorticity scale is critical on the heating augmentation effectiveness. Based on the analysis of Dullenkopf and Mayle ${ }^{(24)}$, the dominant frequency for heating augmentation $f_{\lambda}$ is approximately equal to $0.02 U_{\infty} \delta \delta$ (where $U_{\infty}$ is the post shock velocity). For the 7-inch CEV, $f_{\lambda}$ is between 4 and 20 $\mathrm{kHz}$ and increases with Reynolds number as the boundary layer gets thinner. On the 1.25 -inch CEV, $f_{\lambda}$ is at higher frequencies between 22 and $112 \mathrm{kHz}$. The heat flux PSD measurements on the 0.875 -inch flat face probe and 7-inch CEV show that the energy content at $100 \mathrm{kHz}$ is about 100 times less than at $20 \mathrm{kHz}$, which explains why heating augmentation does not occur on the 1.25-inch CEV. Because the upper $f_{\lambda}$ bound on the 7 -inch model overlaps the lower bound on the 1.25-inch model at about $20 \mathrm{kHz}$, one could expect heating augmentation on the 1.25-inch model when $f_{\lambda} \approx 20 \mathrm{kHz}$. However, this is not the case, because on the 1.25 -inch CEV, $f_{\lambda} \approx 20 \mathrm{kHz}$ corresponds to the lowest Reynolds number $(R e=0.5 E 6 / f t)$ and heating augmentation increases with Reynolds numbers. Assuming a linear variation in the heating augmentation with Reynolds number, one would expect the heating augmentation to decrease by a factor of 5.6 on the smaller model. Since a $10 \%$ augmentation is obtained on the 7-inch CEV at $R e=0.5 E 6 / f t$, the expected augmentation on the 1.25 -inch CEV at that condition is below $2 \%$ which can easily be buried into the experimental systematic and random uncertainties.

Alternatively, one can look at the length scale corresponding to $f_{\lambda}$ which is approximately $16 \delta$. On the 7-inch $\mathrm{CEV}$, this length scale varies from 0.2 to 0.8 inch compared to a variation between 0.028 and 0.15 inch on the smaller model. The dominant length scale on the larger model is similar to the integral length scale of the free stream disturbances whereas on the 1.25 -inch model (with the exception of the $R e=0.5 E 6 / f t$ condition) it is much smaller. This explains the correlation of opposite sensors on the small model. Figure 24 illustrate the difference on how the freestream vorticity interacts with the 7-inch and 1.25-inch models. Disturbance behind the shock layer are clearly seen in Figure 6 a) where the Schlieren image is overlaid on the streamlines (obtained with CFD). It is not obvious if the disturbances are acoustic waves or vortical disturbances. Vortical disturbance can be identified on the Schlieren image. For example, the shear layer from the flow separation over the backshell is clearly visible. Near the stagnation streamline, the disturbances are perpendicular to the streamlines whereas remarkable alignment with the streamlines is seen near the shoulder.

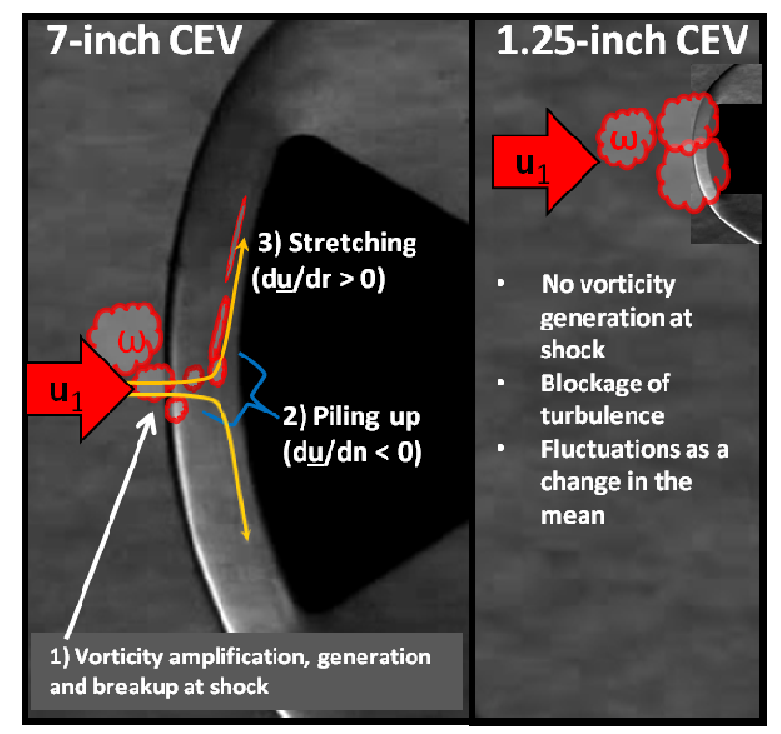

\section{Figure 24. Schematic of the vorticity interaction with the 7-inch and 1.25-inch CEV}

Since multiple pieces of evidence point towards free stream vorticity as the main reason for the respective presence and absence of enhanced heating on large and small models it is imperative to measure or at least estimate the amount of vorticity in the free stream. In tunnel 9, free stream turbulence most likely originates from the particle separator; a perforated conical insert located upstream of the throat used to prevent diaphragm fragments from reaching the test cell. The relatively modest area ratio between the throat and reservoir is such that the flow possesses a significant velocity when it goes through the multiple orifices of the particle separator. By assuming the magnitude of the turbulence intensity at the exit of the particle separator, the free stream turbulence can be estimated using the approximate linear theory of Uberoi ${ }^{(36)}$ or the linear analysis of Ribner and Tucker ${ }^{(37)}$. For a $25 \%$ turbulence intensity at the exit of the particle separator, a turbulence intensity of about $0.25 \%$ is obtained in the free 
stream. As expected, the vorticity is greatly attenuated because of the strong expansion of the Mach 10 flow. The turbulence intensity behind the bow shock can be estimated from Ribner ${ }^{(38)}$

For a Mach 10 flow, the amplification factor based on the pre shock velocity is equal to 1.5 for the lateral component and 1.1 for the streamwise component. Considering the velocity ratio of 5.7 across the shock, the post shock turbulence intensity based on the post shock velocity is approximately equal to $1.7 \%$. The generation of vorticity from the interaction of acoustic waves with the bow shock is another mechanism which could introduce vorticity behind the shock. This mechanism will be considered in the full length paper.

A rough estimate of the heating augmentation with $1.7 \%$ free stream turbulence can be obtained using the data reported by Hoshizaki et al. ${ }^{(17)}$ for spheres in low speed flows. Here, we consider the dimension of the equivalent (untruncated) sphere diameter of the CEV model (2.4D) to evaluate the Reynolds number. At the highest and lowest Reynolds numbers, an augmentation of $70 \%$ and $20 \%$ is obtained which is about twice that measured. This discrepancy might be due to a turbulence level a lower than estimated. The effect of scale size which was not included might also contribute to the discrepancy.

It is outside the scope this paper to analyze the stagnation point heating augmentation measured in other experimental facilities. However, it is nevertheless useful to assess if free stream vorticity is a credible cause for the heating augmentation previously measured in various types of wind tunnels. The turbulent kinetic energy generated in the reservoir of the LaRC 20-Inch Mach 6 Air Tunnel is expected to be less than for Tunnel 9 because to the larger area ratio between the settling chamber and throat. However, less vorticity attenuation will occur during the expansion as the Mach number is less (6 compared to 10). Assuming a Mach number of 0.01 in the settling chamber and using the analysis of Ribner and Tucker (37), the turbulence intensity in the free stream is estimated to be 100 less than that of the settling chamber. A similar ratio was obtained for Tunnel 9. Depending on the turbulence intensity in the reservoir (which is not equivalent to the kinetic energy), similar freestream vorticity levels could be achieved. The amplification of vorticity across the shock will be similar as the effect of Mach number is week at a sufficiently high Mach numbers. In the LaRC 20-Inch Mach 6 tunnel, the generation of vorticity from the interaction of acoustic waves with the bow shock might be more important because of the smaller tunnel size.

In the case of shock tunnels, vorticity can be introduced in the reservoir by the separation of the boundary layer on the shock tube wall when it interacts with the reflected shock. Vorticity generation is expected to increase with stagnation enthalpy as the strength of the reflected shock increases with enthalpy. The amount of vorticity should also be function of the throat to shock tube area ratio where an increase in the shock tube diameter is expected to reduce the amount of vorticity. In the T5 free piston shock tunnel, the Mach number is low at around 5 to maximize the Reynolds number such that the attenuation of vorticity will be less than for higher Mach number tunnels. Therefore, a significant amount of freestream vorticity could be present in the T5 shock tunnel.

It would be useful to compile heating augmentation data from various tunnels and provide estimates of the free stream vorticity based on the geometry of the settling chamber (or shock tube) and nozzle. Better yet would be a direct measurement of the vorticity in the freestream (or settling chamber), but such measurements are difficult to achieve especially because vorticity fluctuations are much less than the acoustic noise in the free stream.

\section{Conclusions}

High frequency pressure and heat transfer measurements combined with the long test time achieve in Tunnel 9 have provided unique statistical data in the stagnation region. The heat transfer augmentation on the 7-inch CEV was found to increase with Reynolds number where it varied from $10 \%$ at $R e=0.5 \mathrm{million} / \mathrm{ft}$ to $40 \%$ at $15 \mathrm{million} / \mathrm{ft}$. On the 1.25-inch CEV, no augmentation was found as very good agreement was obtained with CFD computations. The difference in Reynolds number cannot explain the different heat transfer behavior on the large and small models as both models were at similar $\mathrm{Re}_{\mathrm{D}}$ values.

The increased pressure and heat flux energy at low frequency and greater attenuation at high frequency on the 7inch CEV compared with the smaller probes indicates that the disturbances are modified by the large model. It was determined using space-time correlations that the heat transfer fluctuations at the surface of the 7-inch CEV model are convected at the edge velocity of the boundary layer. Autocorrelation measurements revealed an increase in the longitudinal length scale of the disturbances. Such experimental evidence indicate that the measured heat transfer fluctuations are due to vortical structures which are getting stretched as they move away for the stagnation point. The amplification of free stream vorticity appears to be the dominant mechanism for the heat transfer augmentation on the 7-inch CEV model. On the 1.25-inch model, the correlation between sensors on opposing streamlines indicates that the disturbances are large with respect to the model scale such that vorticity amplification does not occur. The disturbances are then seen as quasi-static variations of the mean flow. This conclusion is reinforced by 
autocorrelation measurements on a 0.875 -inch flat face probe showing the large scale of the incoming disturbances compared to the 1.25-inch model.

The turbulence level in tunnel 9 is estimated to reach $0.25 \%$ in the free stream and $1.7 \%$ behind the bow shock. Because of the high $\mathrm{Re}_{\mathrm{D}}$, this level of turbulence appears sufficient to cause the observed augmentation when compared to experimental data in low speed flow. Improved free stream disturbance measurement with smaller probes will be useful to better understand the nature, intensity and scale of the free stream disturbances. Disturbance measurement in the flow behind the shock layer would also be valuable to understand how much the vorticity is amplified by the shock or created from the interaction of the shock with the acoustic waves.

\section{References}

1. Bertin, J. J. Hypersonic Aerothermodynamics. Washington, DC: American Institute of Aeronautics and Astronautics, 1994.

2. Aeroheating Testing and Predictions for Project Orion CEV at Turbulent Conditions. Hollis, B. R., et al. 2008, AIAA Paper 2008-1226.

3. Hollis, B. R., et al. Experimental Investigation of Project Orion Crew Exploration Vehicle Aeroheating in AEDC Tunnel 9. s.1. : NASA/TP-2008-215547, 2008.

4. Hollis, B.R. Experimental Investigation of Project Orion Crew Exploration Vehicle Aeroheating LaRC 20-Inch Mach 6 Air Tunnel Test 6931. NASA. 2009. NASA/TM-2009-215718.

5. Apollo-Shaped Capsule Boundary Layer Transition at High-Enthalpy in T5. E.C., Marineau, S.J., Laurence and H.G., Hornung. 2010, AIAA Paper 2010-0447.

6. Aeroheating measurements on a reentry capsule model in free-piston shock tunnel HIEST. Tanno, H. et al. s.1. : AIAA Paper 2010-1181, 2010.

7. Heat flux measurement of Apollo capsule model in the free-piston shock tunnel HIEST. Tanno, H. et al. s.1. : AIAA Paper 2009-7304, 2009.

8. Computational Modeling of T5 Laminar and Turbulent Heating Data on Blunt Cones, Part 1: Titan Applications. Olejniczak, J. et al. 2005, AIAA Paper 2005-0176.

9. Heat Flux Calibration of T5 Hypervelocity Shock Tunnel Conical Nozzle in Air. Marineau, E.C and Hornung, H.G. 2009, AIAA Paper 2009-1158.

10. High Enthalpy Cylinder Flow in HEG: A Basis for CFD Validation. Karl, S., Schramm, J.M. and Hannemann, K. 2003, AIAA Paper 2003-4252.

11. Hanamitsu, A., Kishimoto, T. and Bito, H. High Enthalpy Flow Computation and Experiment Around the Simple Bodies. National Aerospace Lab. 1996. NAL-SP 29.

12. Equilibrium Calculation of Augmented Shock-Tunnel Heat Flux at High-Pressure and High-Enthalpy Conditions. Matsukawa, Y. 4, 2011, Journla of Thermophysics and heat Transfer, Vol. 25, pp. 632-634.

13. Studies of Potential Fluid-Mechanical Mechanisms for Enhanced Stagnation-Region Heating. Holden, MS. 1985, AIAA Paper 85-1002.

14. Study of Bow-Shock Wave Unsteadiness in Hypervelocity Flow from Reseveoir Fluctuations. Marineau, E.C. and Horunung, H.G. 2010, AIAA Paper 2010-0382.

15. Hoshizaki, H., et al. Critical Reviw of Stagnation Point Heat Transfer Theory. Air Force flight Dynamics Laboratory. 1975. AFFDL-TR-75-85.

16. Weeks, T. M. Influence of Free-Stream Turbulence on Hypersonic Stagnation Zone Heating. Air Force Flight Dynamis Laboratory. 1968. AFFDL-TR-67-195.

17. Influence of Free-Stream Turbulence on Hypersonic Stagnation Zone Heating. Weeks, T. M. 1969, AIAA Paper 69-167.

18. On the Sensitivity of Heat Transfer in the Stagnation-Point Boundary Layer to Free-Stream Voriticity. Sutera, S. P., Maeder, P. F. and Kesting, J. 4, 1963, Journal of Fluid Mechanics, Vol. 16, pp. 497-520.

19. Analysis of vorticity amplification in the flow approaching a two-dimensional stagnation point. Sadeh, W. Z., Sutera, S.P and Maeder, P. F. 5, 1970, Zeitschrift für Angewandte Mathematik und Physik (ZAMP), , Vol. 21, pp. 699-716.

20. An Analytical Study of Freestream Turbulence Effects on Stagnation Piont Flow and Heat Transfer. Traci, R. M. and Wilcox, D. C. 1974, AIAA Paper 74-515.

21. The distortion of turbulence by a circular cyliner. Britter, R.E., Hunt, J. C. R. and C., Mumford J. 2, Journal of Fluid Mechanics, Vol. 92, pp. 269-301.

22. A theory of turbulent flow round two-dimensional bluff bodies. Hunt, J. C. R. 4, 1972, Journal of Fluid Mechanics, Vol. 61, pp. 625-706. 
23. Stretching of Freestream Turbulence in the Stagnation Region. Wei, C. Y. and J., Miau J. 9, 1992, AIAA Journal, Vol. 30, pp. 2196-2203.

24. Metzger Memorial Session Paper: An Account of Free-Stream-Turbulence Length Scale on Laminar Heat Transfer. Dullenkopf, K. and Mayle, R. E. 3, 1995, Journal of Turbomachinery, Vol. 117, pp. 401-406.

25. Marren, D and Lafferty, J. The AEDC Hypervelocity Wind Tunnel 9. Advanced Hypersonic Test Facilities, Progress in Aeronautics and Astronautics. Reston, VA : American Institute of Aeronautics and Astronautics, 2002, pp. pp. 467-477.

26. Novel Sensor for Fast Heat-Flux Measurements. Knauss, H., et al. 2, 2009, Journal of Spacecraft and Rockets, Vol. 46.

27. Improved Measurements of "Tunnel Noise" Pressure Fluctuations in the AEDC Hypervelocity Wind Tunnel No. 9. Bounitch, A., Lewis, D. and Lafferty, J. 2011, AIAA paper 2011-1200.

28. Two-dimensional Imaging of Soot Volume Fraction in Laminar Diffusion Flames. Snelling, D. R., et al. 12, 1999, Applied Optics, Vol. 38, pp. 2478-2485.

29. Two-Dimensional Soot-Particle Sizing by Time-Resolved Laser-Induced Incandescence. Will, S., Schraml, S. and Leipertz, A. 22, 1995, Vol. 20, pp. $2342-2344$.

30. Boyd, C. F. and Howel, A. Numerical Investigation of One-Dimensional Heat-Flux Calculations. Sliver Spring, MD : s.n., 1994. NSWCDD/TR-94/114.

31. Aerothermal Measurement Improvements Using Coaxial Thermocouples at AEDC Hypervelocity Wind Tunnel No. 9. Coblish, J. J., Coulter, S. M. and Norris, S. D. 2007, AIAA 2007-1467.

32. A Data Parallel Line Relaxation Method for the Navier-Stokes Equations. Wright, M., Bose, D. and Candler, G. 9, AIAA Journal, Vol. 36, pp. 1603-1609.

33. A review of velocity and pressure fluctuations in turbulent flows around bluff bodies. Hunt, J. C. R. et al. 1990, Journal of Wind Engineering and Industrial Aerodynamics, Vol. 35.

34. On surface pressure fluctuations beneath turbulent flow round bluff bodies. Durbin, P. A. and R., Hunt J. C. 1, 1980, Journal of Fluid Mechanics, Vol. 100, pp. 161-184.

35. Characteristics of pressure fluctuations on the windward wall of a tall building. Kawai, H., Katsura, J. and Ishizaki, H. 1980. Proc. 5th. Conf. on Wind Engineering. pp. 519-528.

36. Effect of Wind-Tunnel Contraction on Free-Stream Turbulence. Uberoi, M. S. 1956, Journal of the Aeronautical Sciences, pp. 754-764.

37. Ribner, H. S. and Tucker, M. Spectrum of turbulence in a contracting stream. NACA. 1953. NACA-TR-1113. 38. Spectra of noise and amplified turbulence emanating from shock-turbulence interaction. Ribner, H. S. 1987, AIAA Journal, Vol. 25, pp. 436-442. 